\title{
Change in Brain Plasmalogen Composition by Exposure to Prenatal Undernutrition Leads to Behavioral Impairment of Rats
}

\author{
Kodai Hino, ${ }^{1}$ Shunya Kaneko, ${ }^{1}$ Toshiya Harasawa, ${ }^{1}$ Tomoko Kimura, ${ }^{1}$ Shiro Takei, ${ }^{2}$ Masakazu Shinohara,, 3 \\ Fumiyoshi Yamazaki, ${ }^{5}{ }^{\circledR}$ Shin-ya Morita, ${ }^{6}$ Shumpei Sato, ${ }^{5}$ Yoshihito Kubo, ${ }^{1}$ Tadaaki Kono, ${ }^{1}{ }^{\circledR}$ Mitsutoshi Setou, ${ }^{5,7,8}$ \\ Mina Yoshioka, ${ }^{1}$ ○ Junya Fujino, ${ }^{1}$ Hiroyuki Sugihara, ${ }^{9}$ Hideto Kojima, ${ }^{10}$ Naoto Yamada, ${ }^{11}$ and ${ }^{\circ}$ Jun Udagawa ${ }^{1}$ \\ ${ }^{1}$ Division of Anatomy and Cell Biology, Department of Anatomy, Shiga University of Medical Science, Otsu 520-2192, Japan, ${ }^{2}$ Department of Environmental \\ Biology, College of Bioscience and Biotechnology, Chubu University, Kasugai 487-8501, Japan, ${ }^{3}$ Division of Epidemiology, Kobe University Graduate School \\ of Medicine, Kobe 650-0017, Japan, ${ }^{4}$ Integrated Center for Mass Spectrometry, Kobe University Graduate School of Medicine, Kobe 650-0017, Japan, \\ ${ }^{5}$ International Mass Imaging Center and Department of Cellular and Molecular Anatomy, Hamamatsu University School of Medicine, Shizuoka 431-3192, \\ Japan, ${ }^{6}$ Department of Pharmacy, Shiga University of Medical Science Hospital, Shiga 520-2192, Japan, ${ }^{7}$ Preeminent Medical Photonics Education \& \\ Research Center, Shizuoka 431-3192, Japan, ${ }^{8}$ Department of Anatomy, University of Hong Kong, Pokfulam, Hong Kong SAR, China, ${ }^{9}$ Division of Molecular \\ Diagnostic Pathology, Department of Pathology, Shiga University of Medical Science, Otsu 520-2192, Japan, ${ }^{10}$ Department of Stem Cell Biology and \\ Regenerative Medicine, Shiga University of Medical Science, Otsu 520-2192, Japan, and ${ }^{11}$ Department of Psychiatry, Shiga University of Medical Science, Otsu \\ 520-2192, Japan
}

Epidemiological studies suggest that poor nutrition during pregnancy influences offspring predisposition to experience developmental and psychiatric disorders. Animal studies have shown that maternal undernutrition leads to behavioral impairment, which is linked to alterations in monoaminergic systems and inflammation in the brain. In this study, we focused on the ethanolamine plasmalogen of the brain as a possible contributor to behavioral disturbances observed in offspring exposed to maternal undernutrition. Maternal food or protein restriction between gestational day (GD) 5.5 and GD 10.5 resulted in hyperactivity of rat male adult offspring. Genes related to the phospholipid biosynthesis were found to be activated in the PFC, but not in the NAcc or striatum, in the offspring exposed to prenatal undernutrition. Corresponding to these gene activations, increased ethanolamine plasmalogen (18:0p-22:6) was observed in the PFC using mass spectrometry imaging. A high number of crossings and the long time spent in the center area were observed in the offspring exposed to prenatal undernutrition and were mimicked in adult rats via the intravenous injection of ethanolamine plasmalogen (18:0p22:6) incorporated into the liposome. Additionally, plasmalogen (18:0p-22:6) increased only in the PFC, and not in the NAcc or striatum. These results suggest that brain plasmalogen is one of the key molecules to control behavior, and its injection using liposome is a potential therapeutic approach for cognitive impairment.

Key words: hyperactivity; maternal undernutrition; plasmalogen phosphatidylethanolamine; prefrontal cortex

Significance Statement

Maternal undernutrition correlates to developmental and psychiatric disorders. Here, we found that maternal undernutrition in early pregnancy led to hyperactivity in rat male offspring and induced gene activation of phospholipid-synthesizing enzyme and elevation of ethanolamine plasmalogen (18:0p-22:6) level in the PFC. Intravenous injection of ethanolamine plasmalogen (18:0p-22:6) incorporated into the liposome maintained crossing activity and the activity was circumscribed to the center area for a long time period, as in prenatally undernourished offspring with aberrant behavior. Furthermore, the amount of ethanolamine plasmalogen (18:0p-22:6) increased in the PFC of the rat after injection. Our result suggests that brain plasmalogen is one of the key molecules to control behavior and that its injection using liposome is a potential therapeutic approach for cognitive impairment. 


\section{Introduction}

Epidemiological studies have linked maternal stress during pregnancy, including malnutrition, infection, daily life stress, and traumatic events, to the presence of psychological and developmental disorders in offspring (Hoek et al., 1998; Khashan et al., 2008; Kinney et al., 2008; Marques et al., 2015; Fineberg et al., 2016; Kundakovic and Jaric, 2017). A previous study suggested that brain development is disrupted by prenatal exposure to stress, which alters fetal programming by affecting the epigenome, such as via changes to DNA methylation and histone modification, and induces behavioral disturbances (Kundakovic and Jaric, 2017). A postmortem study of the brain of a patient with schizophrenia suggested decreased Reelin and GAD67 expression due to the hypermethylation of their promoter regions, which was led by the upregulation of DNA methyltransferase 1 (DNMT1) genes and may be involved in the etiology of schizophrenia (Kundakovic, 2014). Meanwhile, a Dutch famine study reported the relationship between prenatal undernutrition during the first trimester and the increased incidence of schizophrenia (Brown and Susser, 2008). These reports suggest that maternal stress may alter brain function through disturbances in the neurotransmission of certain systems, such as the GABAergic system. Indeed, restraint stress on mice during pregnancy leads to the overexpression of DNMT1 and DNMT3a mRNA, which is accompanied by the downregulation of Reelin and GAD67 protein levels, as well as glutamine receptor protein from the hypermethylation of their promoter regions in the frontal cortex. This cascade of events induced a schizophrenia-like phenotype observable in behavioral tests performed on the male offspring after birth (Matrisciano et al., 2013). Prenatal stress is thus associated with a predisposition toward neurobehavioral disorders. Not only restrained stress, but prenatal caloric restriction has also been shown to affect the dopamine system and neuronal excitability, resulting in a decrease in anxiety-like behavior, while protein restriction results in deficits in prepulse inhibition and locomotor activity (Markham and Koenig, 2011; Amaral et al., 2015). Based on these findings, various neurotransmitter systems, including monoamine, GABAergic, and glutaminergic systems, appear to be viable therapeutic targets for treating behavioral disturbances induced by prenatal stress; however, membrane lipids also seemed to differ between the brains of patients with psychiatric disorders and those without such disorders, and little is known regarding the underlying mechanisms of this process (Ghosh et al., 2017). Therefore, we hypothesized that

Received Oct. 21, 2018; revised July 28, 2019; accepted July 31, 2019.

Author contributions: K.H., S.K., S.T., and J.U. designed research; K.H., S.K., T.H., T. Kimura, S.T., M. Shinohara, F.Y., S.-y.M., Y.K., T. Kono, M.Y., J.F., and J.U. performed research; K.H., S.K., T.H., T. Kimura, S.T., M. Shinohara, F.Y., S.-y.M., Y.K., T. Kono, M. Setou, H.S., H.K., N.Y., and J.U. contributed unpublished reagents/analytic tools; K.H., S.K., T.H., T. Kimura, S.T., M. Shinohara, F.Y., S.-y.M., S.S., Y.K., T. Kono, M. Setou, and J.U. analyzed data; K.H., S.T., S.-y.M., S.S., M. Setou, H.S., and J.U. edited the paper; K.H. and J.U. wrote the paper; M. Shinohara and J.U. wrote the first draft of the paper.

This work was supported by Japan Society for the Promotion of Science KAKENHI Grants JP 25460242 and $16 K 08441$ to J.U., Food Science Institute Foundation (Ryoushoku-kenkyukai) to J.U., SUMS President's Grant for Encouragement of Young Researchers Grant $1515503 Z$ Z to K.H., SUMS grant for the special research project Grant $1515503 Z Z E$ to J.U., SUMS grant for the integration of basic and clinical research Grant $1515503 Z 0$ to J.U., Ministry of Education, Culture, Sports, Science and Technology/Japan Society for the Promotion of Science KAKENHI Grant JP15H05898B1 to M. Setou, and Ministry of Education, Culture, Sports, Science and Technology (Imaging Platform) to M. Setou. F.Y. was supported by Ministry of Education, Culture, Sports, Science and Technology (Imaging Platform). MALDI-IMS was conducted via the Imaging Platform supported by the Ministry of Education, Culture, Sports, Science and Technology, Japan, and Preppers Co. Ltd. We thank Mr. Kenji Iwabuchi (Department of Stem Cell Biology and Regenerative Medicine, Shiga University of Medical Science) for assistance in the behavioral test.

The authors declare no competing financial interests.

Correspondence should be addressed to Jun Udagawa at udagawa@belle.shiga-med.ac.jp.

https://doi.org/10.1523/JNEUROSCI.2721-18.2019

Copyright $\odot 2019$ the authors behavioral disturbance due to early prenatal undernutrition is led by aberrant brain phospholipid metabolism via fetal programming. In this study, we focused on alterations in the composition of brain phospholipids that are induced by prenatal undernutrition, and we have identified a candidate phospholipid to control behavior.

\section{Materials and Methods}

Animals

All animal procedures were approved by the Institutional Review Board of the Shiga University of Medical Science Animal Care and Use Committee (2011-8-1, 2014-3-7, 2015-12-1, and 2019-4-2). For experiments investigating maternal undernutrition, 9-week-old male (body weight [BW], 250-280 g) and 8-week-old female (BW, 160-190 g) Wistar rats were obtained from CLEA Japan. Six-week-old male rats were obtained for experiments involving phosphatidylethanolamine (PE) injection. All rats were housed under a $12 \mathrm{~h}$ light/dark cycle (lights were turned on at 08:00) and were allowed to acclimate for $>1$ week.

\section{Diet}

Female rats were acclimated to a standard diet for pregnant rats (AIN$93 \mathrm{G}$ ), containing $20 \%$ casein for $2 \mathrm{~d}$ before mating, for which each female was housed with 1 male overnight. We defined gestational day (GD) 0 as the day when a vaginal plug was observed. Pregnant rats were randomly assigned to the ad libitum (AL) group, the food-restriction (F) group, or the isocaloric, low-protein diet (LPD) group, and subjected to undernutrition from GD5.5 to GD10.5 or from the day of blastocyst implantation to the day just before the closure of the neural tube (Fig. 1A) (Erb, 2006). In humans, this period is comparable to the days from E6.5 to approximately E30, which is nearing the first half of the first trimester (Bystron et al., 2008; Schoenwolf et al., 2015). Neural stem cells, and not neurons, exist in the telencephalon because this period is before neurogenesis (Götz and Huttner, 2005; Bystron et al., 2008). The F group was fed 50\% (50F, four dams) or $40 \%$ (40F, six dams) of the daily food intake of the $\mathrm{AL}$ group (eight dams). The rationale is that daily rations fell to no more than 800 kcal during the Dutch famine that occurred between December 1944 and April 1945, representing $40 \%$ of rations (>2000 calories) after June 1945 (Roseboom et al., 2001). The LPD group (five dams) was fed a diet containing 9\% casein. After delivery, pups were culled to produce litters of eight offspring ( 4 males and 4 females) per a dam on postnatal day (P)4. During lactation, the dams were fed CE-2, a standard pellet chow for rearing and breeding. Subsequently, the offspring were weaned on P28; and afterward, they were fed CE-2 ad libitum. Male offspring were used in this study because male humans show a higher risk of neuropsychiatric or neurobehavioral disorders (e.g., schizophrenia, attention deficit hyperactivity disorder $[\mathrm{ADHD}]$, and autism spectrum disorder [ASD]), than females (Aleman et al., 2003; Werling and Geschwind, 2013; Arnett et al., 2015). Furthermore, sex differences in ADHD and ASD may be, in part, genetically mediated (Werling and Geschwind, 2013; Arnett et al., 2015). The male offspring experienced handling once a week after weaning, and BW of the offspring were measured at 9 and 12 weeks of age.

\section{Preparation of liposomes}

Large unilamellar liposomes composed of egg phosphatidylcholine (PC) and C18:0-22:6 plasmalogen PE (PlsEtn) or C16:0-18:1 diacyl PE (POPE) were prepared by the extrusion method (Morita et al., 2008). Briefly, a thin film was obtained by evaporating the lipid chloroform solution and was subsequently hydrated with saline so that the concentrations of egg PC and PE (18:0p-22:6) (Avanti) were $8 \mathrm{mg} / \mathrm{ml}$ and 2 $\mathrm{mg} / \mathrm{ml}$, respectively, for PE liposomes (PELs). Similarly, mixed solutions of $\mathrm{PE}(16: 0-18: 1)$ and egg $\mathrm{PC}$ were prepared at the same concentration as for the POPE liposomes (POPELs). To produce control liposomes (CLs), PC $(10 \mathrm{mg} / \mathrm{ml})$ with no PE was prepared. After five rounds of freezing and thawing, the lipid suspension was extruded through a polycarbonate filter with $100 \mathrm{~nm}$ pore size.

\section{Behavioral test}

Effects of prenatal undernutrition on behavior. From June to October 2012 to 2014 , the locomotor activity of the male offspring was evaluated by the 

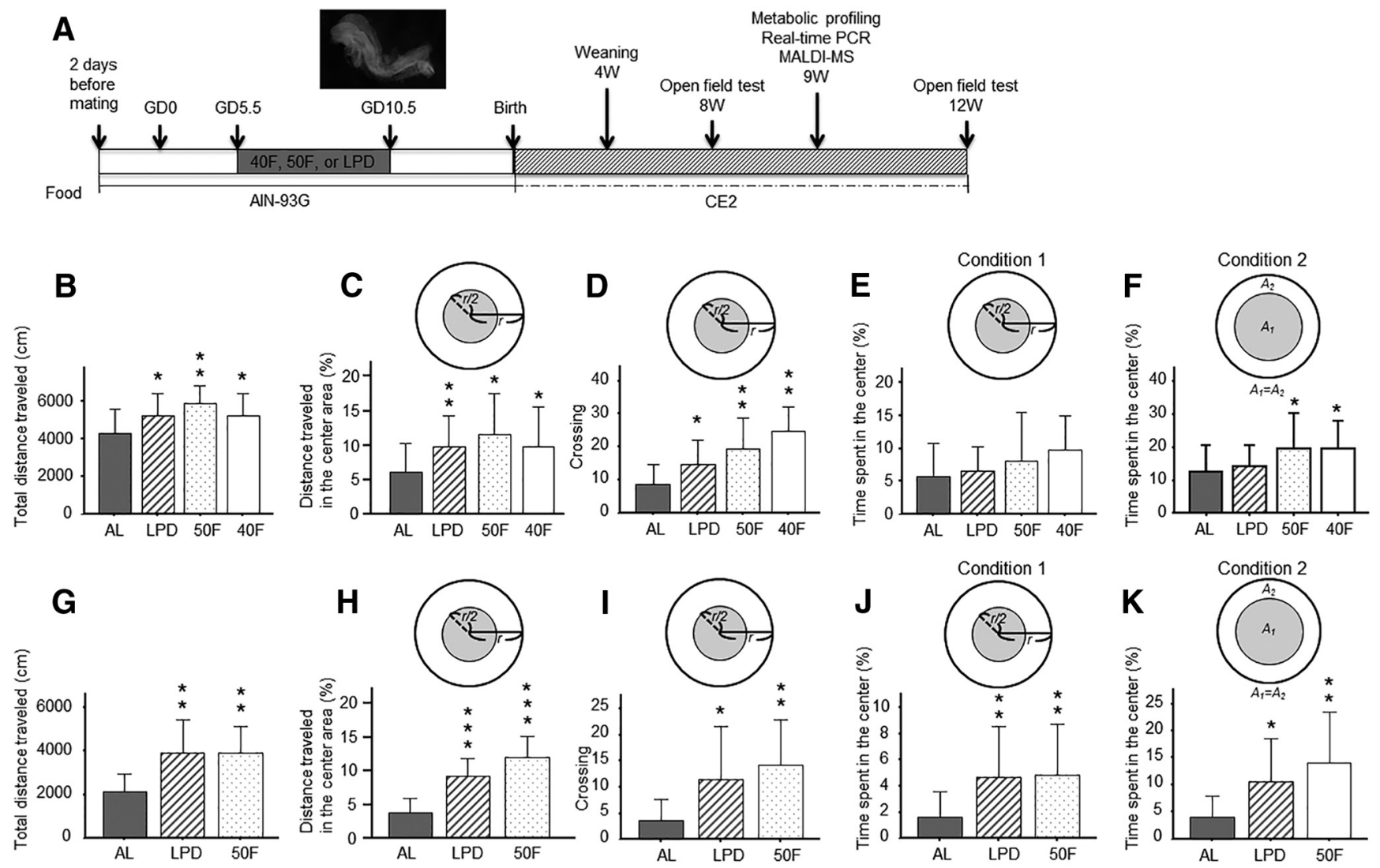

Figure 1. Study design and behavior of rat offspring subjected to prenatal undernutrition. $A$, The experimental schedule is shown. The daily food intake was restricted from GD $5.5-10.5$ or from the day of blastocyst implantation to the day just before the closure of the neural tube. $B-F$, Behavioral tests at 8 weeks of age: male rat offspring exhibited hyperactivity in the LPD ( $n=18$ ), $50 \mathrm{~F}$ ( $n=13)$, and $40 \mathrm{~F}(n=17)$ groups compared with the offspring in the AL group $(n=32)$. $\mathbf{G}-\boldsymbol{K}$, Behavioral tests at 12 weeks of age: behavioral disturbances continued at 12 weeks of age in the $L P D$ $(n=19)$ and $50 \mathrm{~F}(n=14)$ offspring compared with the AL offspring $(n=18)$. One-way ANOVA with Dunnett's test: ${ }^{*} p<0.05 ;{ }^{* *} p<0.01 ;{ }^{* * *} p<0.001$. A1, Area of the center region; A2, area of the peripheral region.

open-field test at 8 weeks of age for the AL ( $n=32$ from 8 dams), LPD ( $n=18$ from 5 dams), $50 \mathrm{~F}$ ( $n=13$ from 4 dams), and $40 \mathrm{~F}(n=17$ from 6 dams) groups and at 12 weeks for the $\mathrm{AL}$ ( $n=18$ from 5 dams), LPD ( $n=19$ from 5 dams), and $50 \mathrm{~F}$ ( $n=14$ from 5 dams) groups, to examine the impact of maternal undernutrition on behavior. Behavioral data that included device errors in tracing animals were excluded. The apparatus, measuring $90 \mathrm{~cm}$ in diameter and $45 \mathrm{~cm}$ in height, was used to monitor the behavior of rats, and the behavior was recorded for $10 \mathrm{~min}<9$ lux of light. Data were analyzed using the Limelight video tracking system (Actimetrics). The distance traveled and the time spent in the center were measured under the following analysis conditions: the open field was divided into the center and peripheral regions so that (1) the center region was bordered by a concentric circle passing through the midpoint of the radius of the open field (Condition 1 and 2) and the area of the center region (A1) was the same as that of the peripheral region (A2) (Condition 2). The former condition was selected to allow crossing behavior to be analyzed.

Effects of plasmalogen (18:0p-22:6) on behavior. To examine the effect of PE (18:0p-22:6), the locomotor activity of male rats was evaluated before and after PE (18:0p-22:6) injection (for 8- and 14-week-old offspring, respectively). Rats were assigned to two different groups based on the results of crossing from the open-field test so that rats with similar locomotor activities were evenly divided among the groups. A PEL or CL suspension $(1 \mathrm{ml} / \mathrm{kg} \mathrm{BW})$ was injected into the tail vein at 14 weeks of age, and the second injection of liposome suspension was performed $2 \mathrm{~d}$ later. The rats in the PEL $(n=6)$ and CL $(n=7)$ groups were subjected to the open-field test or the elevated plus maze test (PEL: $n=6$, CL: $n=6) 1$ or $4 \mathrm{~d}$ after the second injection, respectively. Behavioral data of 1 CL rat acquired by using the elevated plus maze test was excluded because it included device errors in tracing animals. In the elevated plus maze test,
Table 1. Allocation of experimental groups

\begin{tabular}{llcl}
\hline Treatment & Experiment & Age (weeks) & \\
\hline Undernutrition & Open-field test & 8 & AL, 40F, 50F, LPD \\
& Open-field test & 12 & AL, 50F, LPD \\
& Metabolic profiling (plasma, CSF) & 9 & AL, 40F \\
& Counting microglial cells (PFC) & 9 & AL, 40F \\
& Gene expression (PFC, NAcc, CPu) & 9 & AL, 40F \\
& MALDI-IMS (PFC, NAcc, CPu) & 9 & AL, 40F \\
Liposome injection & Open-field test & 8 and 14 & CL, PEL, POPEL, saline \\
& Elevated plus maze test & 8 and 14 & CL, PEL, POPEL, saline \\
& MALDI-IMS (PFC, NAcc, (Pu, blood cells) & 14 & CL, PEL \\
\hline
\end{tabular}

rats were placed in the central square platform facing the closed arms, and their behavior was recorded for $250 \mathrm{~s}<8,10$, and 4 lux of light at the central square platform, facing the open arms and closed arms, respectively (Hino et al., 2019). Time spent in the open and closed arms was measured in this test. To verify the specific effect of PlsEtn (18:0p-22:6) on behavior, the alteration of locomotor activity was examined before and after POPEL, CL, or saline injection. Male rats were assigned to three different groups based on the results of the crossing analysis, and behavior was evaluated for POPEL $(n=5)$, CL $(n=5)$, and saline injection $(n=4)$ groups. This experiment was conducted separately from the PEL injection study. The experimental groups allocated in this study are listed in Table 1.

\section{Metabolic profiling of plasma and CSF}

Blood samples were collected from male offspring (AL: four dams and seven litters; 40F: four dams and six litters) and CSF (AL: four dams and seven litters; 40F: four dams and five litters) at 9 weeks of age. CSF 
samples that got mixed with blood were excluded from the analysis. Rats were anesthetized with sodium pentobarbital solution $(35 \mathrm{mg} / \mathrm{kg}$, i.p.) during the light phase (16:00-18:00). They were placed in a stereotaxic device (Kopf Instruments), and $50 \mu \mathrm{l}$ of CSF was collected immediately from the cisterna magna. Then, after decapitation, $5 \mathrm{ml}$ of blood was collected in test tubes containing EDTA-2Na. The plasma was collected after centrifugation of the blood at $2000 \times g$ for $15 \mathrm{~min}$. Samples were stored at $-80^{\circ} \mathrm{C}$ until use.

Hydrophilic metabolites were extracted using the $\mathrm{MeOH}-\mathrm{CHCl}_{3}$ method according to the procedure detailed in previous reports (Tsugawa et al., 2011; Nishiumi et al., 2012). Fifty microliters of plasma or CSF was mixed with $250 \mu \mathrm{l}$ of a solvent mixture $\left(\mathrm{MeOH}: \mathrm{H}_{2} \mathrm{O}: \mathrm{CHCl}_{3}\right.$, 2.5:1:1, v/v/v) containing $20 \mu \mathrm{l}$ of $0.25 \mathrm{mg} / \mathrm{ml} 2$-isopropylmalic acid (Sigma-Aldrich) as the internal standard. The mixture was then shaken at $37^{\circ} \mathrm{C}$ for $30 \mathrm{~min}$ and centrifuged at $16,000 \times g$ for $5 \mathrm{~min}$ at $4^{\circ} \mathrm{C}$. Then, 225 $\mu \mathrm{l}$ of supernatant was mixed with $200 \mu \mathrm{l}$ of distilled water, and the solution was centrifuged at $16,000 \times g$ for $5 \mathrm{~min}$ at $4^{\circ} \mathrm{C}$. The resultant supernatant $(250 \mu \mathrm{l})$ containing hydrophilic primary metabolites was collected and lyophilized using a freeze dryer. For oximation, $40 \mu \mathrm{l}$ of 20 $\mathrm{mg} / \mathrm{ml}$ methoxyamine hydrochloride (Sigma-Aldrich) dissolved in pyridine was mixed with a lyophilized sample, and the mixture was then shaken at $30^{\circ} \mathrm{C}$ for $90 \mathrm{~min}$. For derivation, $20 \mu \mathrm{l}$ of $\mathrm{N}$-methyl-Ntrimethylsilyl-trifluoroacetamide (GL Science) was added, and the mixture was shaken at $37^{\circ} \mathrm{C}$ for $30 \mathrm{~min}$. The mixture was then centrifuged at $16,000 \times g$ for $5 \mathrm{~min}$ at $4^{\circ} \mathrm{C}$, and the resultant supernatant was subjected to gas chromatography-mass spectrometry analysis.

Gas chromatography-mass spectrometry analysis was performed by using a GCMS-QP2010 Ultra device (Shimadzu) with a fused-silica capillary column (CP-SIL $8 \mathrm{CB}$ low bleed/MS; $30 \mathrm{~m} \times 0.25 \mathrm{~mm}$ inner diameter, film thickness: $0.25 \mu \mathrm{m}$; Agilent Technologies). The front inlet temperature was set at $230^{\circ} \mathrm{C}$. The flow rate of helium gas through the column was $39.0 \mathrm{~cm} / \mathrm{s}$. The column temperature was held at $80^{\circ} \mathrm{C}$ for $2 \mathrm{~min}$ and then raised by $15^{\circ} \mathrm{C} / \mathrm{min}$ to $330^{\circ} \mathrm{C}$ and held for $6 \mathrm{~min}$. The transfer-line and ion-source temperatures were $250^{\circ} \mathrm{C}$ and $200^{\circ} \mathrm{C}$, respectively. Twenty scans per second were recorded over the mass range of $85-500 \mathrm{~m} / \mathrm{z}$ by using the Advanced Scanning Speed Protocol (Shimadzu).

Raw data were exported in netCDF format, and peak detection and alignment were performed by using MetAlign software (Wageningen University \& Research). The resulting data were exported in CSV format and then analyzed with in-house analytical software (AIoutput), which enabled peak identification and semiquantification by using an in-house metabolite library (Tsugawa et al., 2011; Nishiumi et al., 2012). For semiquantification, the peak height of a particular ion for each metabolite was normalized to the peak height of the specified ion of 2-isopropylmalic acid (the internal standard).

\section{Brain sections}

According to previous reports, lesions in the medial PFC cause alterations in the locomotor activity of rats (Jinks and McGregor, 1997; Fritts et al., 1998). Moreover, the NAcc and striatum (caudate putamen [CPu]) receive input from the PFC and are associated with locomotor activity and impulsivity (Moreno et al., 2013; Spencer et al., 2015; Scofield et al., 2016; Zhu et al., 2016; Dahoun et al., 2017). Hence, sections of PFC, $\mathrm{NAcc}$, and $\mathrm{CPu}$ were subjected to gene expression and phospholipid analyses, and immunohistochemistry. Male offspring of 9 weeks of age from the maternal undernutrition experiment and male rats injected with PEL or CL at 14 weeks of age (at $10 \mathrm{~h}$ after the elevated plus maze test) were anesthetized and killed with sodium pentobarbital solution (100 mg/kg, i.p.). Brain samples from the AL (four dams and eight litters), $40 \mathrm{~F}$ (five dams and eight litters), PEL $(n=4)$, and CL $(n=4)$ groups were immediately dissected out and frozen in dry ice. Cryosections of the brain were cut at a thickness of $10 \mu \mathrm{m}$ before use for gene expression and phospholipid analyses. Greater than eight sections of the PFC every $200 \mu \mathrm{m}$, greater than six sections of NAcc every $80 \mu \mathrm{m}$, and greater than seven sections of CPu every $80 \mu \mathrm{m}$ were placed on Platinum Pro (Matsunami) or polyethylene naphthalate membrane slides (Leica Microsystems) for immunohistochemistry or gene expression analysis, respectively. Sections at 0.8 and $2 \mathrm{~mm}$ from the frontal end of the cerebral cortex, a section at $0.32 \mathrm{~mm}$ from the anterior end of the NAcc, and a section at $0.48 \mathrm{~mm}$ from the anterior end of the $\mathrm{CPu}$ were placed on indium tin oxide-coated glass slides (Bruker Daltronics) for phospholipid analysis.

\section{Gene expression}

The PFC, NAcc, and $\mathrm{CPu}$ of male offspring in the $\mathrm{AL}$ and $40 \mathrm{~F}$ groups were dissected and collected from brain sections by a laser microdissection system (LMD6000, Leica Microsystems). Gene expression analysis was performed according to the protocol described in the previous report (Kimura et al., 2018). Isolated total RNA was converted to cDNA via reverse transcription (RT) and amplified using the Ovation PicoSL WTA system V2 (NuGEN Technologies). The mRNA expression levels were estimated using qRT-PCR analysis using a LightCycler 480 system (Roche Diagnostics) with SYBR Premix Ex TaqII polymerase (Takara). The qRT-PCR was performed in duplicates, and comparative $\mathrm{C}_{\mathrm{q}}$ values of the target genes (Table 2) normalized to $\mathrm{B} 2 \mathrm{~m}$, a reference gene, were compared between the $\mathrm{AL}$ and $40 \mathrm{~F}$ groups.

\section{Counting microglial cells and activated microglial cells in PFC}

In each rat, 10 or more brain sections at $\sim 4.8-2.6$ rostral to the bregma were fixed with $4 \%$ PFA at room temperature for $30 \mathrm{~min}$, incubated with rabbit anti-Iba1 antibody (dilution, 1:500; Wako, catalog \#019-19741, RRID:AB_839504) and mouse anti-CD11b (dilution, 1:300; Bio-Rad/ AbD Serotec, catalog \#MCA275R, RRID:AB_321302), and then incubated with goat anti-rabbit IgG (H\&L) conjugated with DyLight 488 (dilution, 1:500; Abcam, catalog \#ab96895, RRID:AB_10679405) and goat anti-mouse IgG (H\&L) conjugated with DyLight 549 (dilution, 1:500; Rockland, catalog \#610-142-121, RRID:AB_1057533). Sections were stained with DAPI, and the numbers of all Ibal-expressing microglial cells and CD11b-labeled activated microglial cells were counted in the medial PFC by using a fluorescence microscope (IX83, Olympus). The density of those cells was then compared between offspring in the AL and $40 \mathrm{~F}$ groups.

\section{Matrix-assisted laser desorption/ionization-imaging mass spectrometry (MALDI-IMS)}

MALDI-IMS was performed by using the PFC, NAcc, and CPu samples in both experiments as previously described (Hossen et al., 2015; Sugiyama et al., 2015). Tissues on indium tin oxide-coated glass slides were subjected to matrix application by the sublimation/deposition method, with $1 \mathrm{~g}$ of 9 -aminoacridine sublimated at $210^{\circ} \mathrm{C}$ in order for the deposition thickness to reach $1.0 \mu \mathrm{m}$ by using the iMLayer device (Shimadzu). Experiments were performed by using a mass microscope, a prototype of the iMScope equipped with a $355 \mathrm{~nm} \mathrm{Nd:YAG} \mathrm{laser} \mathrm{(Shi-}$ madzu). Negative ions from a sample area of $30 \mu \mathrm{m} \times 30 \mu \mathrm{m}$ on the PFC, $\mathrm{NAcc}$, and $\mathrm{CPu}$ samples were obtained in a mass range of $\mathrm{m} / \mathrm{z} 400$ to 1000. Adjacent sections of a mouse brain as a reference were laid together with rat brain sections of $40 \mathrm{~F}$ and $\mathrm{AL}$ offspring, and the rats injected with PEL and CL and were used to correct for differences in peak intensity due to differences in sample preparation between slides (Fig. 2). The peaks of $52 \mathrm{PE}, 16$ phosphatidylserine (PS), 14 phosphatidylinositol (PI), 10 lyso$\mathrm{PE}$, and 4 lysophosphatidylinositol (lyso-PI) were detected (Taguchi and Ishikawa, 2010). The peak intensity of each individual phospholipid was corrected using the average intensity of the corresponding phospholipid of the mouse references. The average peak intensities of the PFC, NAcc, and $\mathrm{CPu}$, respectively, were compared between the $\mathrm{AL}$ and $40 \mathrm{~F}$ groups and between the PEL and CL groups.

To identify the peak assigned at $\mathrm{m} / \mathrm{z} 774.5$ as the peak of PE (18:0p-22: 6 ), we performed MALDI tandem mass spectrometry using the mass microscope described above (Sugiyama et al., 2015) and identified PE (18:0p-22:6) via collision-induced dissociation (Zemski Berry et al., 2014).

\section{Blood cells}

Blood was collected from 14-week-old male rats for the PEL and CL injection experiment. Whole blood cells were washed with saline, applied to a 12-well flexiPERM plate (Sarstedt), and affixed to an indium tin oxide-coated glass slide (Matsunami Glass Industries), of which the surface was coated with poly-L-lysine (Hossen et al., 2015) to ensure that the 
Table 2. Primer sequences for real-time PCR

\begin{tabular}{|c|c|c|c|c|}
\hline Gene symbol & Forward primer sequence $\left(5^{\prime}>3^{\prime}\right)$ & Reverse primer sequence $\left(5^{\prime}>3^{\prime}\right)$ & Ascension number & Amplicon size (bp) \\
\hline Agps & TCCTACTCACAAGACGCAGA & AGGAATCCGCTCAAACATCC & NM_053350.2 & 99 \\
\hline Apoe & CCGCAACGAGGTAAACACCA & ATCATCCGCATCCCGCATC & NM_001270684.1 & 109 \\
\hline Aqp9 & AGCCGGATAGCGAAGGAGA & AGTGATGATCCCGCCAAAAC & NM_022960.2 & 120 \\
\hline Cds2 & CCACCGGTTCATCTCCTTTAC & GCGTTACGACAATAAGCAAGG & NM_053643.1 & 134 \\
\hline Cept1 & AGTCTTCTACTGCCCTACAGC & TTCTTCTGGCCTGTTTCCCG & NM_001007.1 & 119 \\
\hline Chka & CAACAACTGCACAAGTTCCTC & CTCTTGGCCTTCCAACAATAAG & NM_017127.1 & 144 \\
\hline Far1 & сCCTTGCAGATCTCGTTCCT & AGGATTAGTGCTGCCTGTTGT & XM_006230020.3 & 125 \\
\hline$G k$ & GAAACTTCGTTGGCTCCTCG & GAACACCGCCATTGATTCCC & NM_024381.2 & 128 \\
\hline Gnpat & GTGTGTGTGAATGAAGAAGGCA & GGACAAAGGACAGCATGAGGA & NM_053410.1 & 115 \\
\hline Gpat1 & GGCAACAACCTCAACATCCC & TTGCGTCCATCTGGAGTTTC & NM_017274.1 & 98 \\
\hline Gpat3 & ACACTGGTTGGCCAGCTTC & AGACAGGGAGCGAACACAGA & XM_008770021.2 & 96 \\
\hline Gpat4 & GCTCAAACCAGACATGGGGG & TTGCTAACCATACGTCGCCC & NM_001047849.1 & 141 \\
\hline Mfsd $2 a$ & GCTTCTGCATCAGCAAGTCC & GGGAAGTCAGGCACAAACCA & NM_001106683.1 & 118 \\
\hline Mfsd3 & GGTGCTTCTGCCTCAGATTT & GCACTGACCTCAACAGCTTC & NM_001024908.1 & 142 \\
\hline Plpp4 & TGCTTTCCAGATGGGGTGATG & CAACTTGCCAGCCAGGTAGAA & NM_001191631.1 & 150 \\
\hline Pcyt1a & ATCCCCTACTCTTCGGCAGG & GGACAATGCGGGTGATGAGG & NM_078622.2 & 121 \\
\hline Pcytib & TCATCTCGGGGTTCTGATGAC & ACGGACAATTCTGGTGATGATG & NM_173151.1 & 114 \\
\hline Pcyt2 & ATGTGGCTGGTGCCTTTGA & AAAGTGTAGGCCCGCGATGA & NM_053568.1 & 104 \\
\hline Pemt & GTACTGGGGAAGTACAGCCAAC & CTTCAAACAGGAGAGCAACCAC & NM_013003.1 & 116 \\
\hline Pfkp & AAGTACCTGGAGCACCTCTCT & TGTATATTCCCATGCGCACCA & NM_206847.1 & 115 \\
\hline Pisd & ATGTGGGCTCTATCCGCATC & TGGGAATGCCCTCCTTGTTG & XM_002725000.4 & 120 \\
\hline Ptdss1 & TATGGGCTCTGCTGGACAATC & CCACCACCATTACACAACAGG & NM_001012133.1 & 131 \\
\hline Ptdss2 & AACCCCTCAGGATACAGCCTAC & CTAACACACAGCCAAAACCGC & NM_001106316.1 & 144 \\
\hline Smpd3 & TGTCTCAACAGGGGTCTCTTC & GTACAGGCGATGTACCCAACA & NM_053605.1 & 176 \\
\hline
\end{tabular}

A

Indium tin oxide (ITO)-coated glass slide

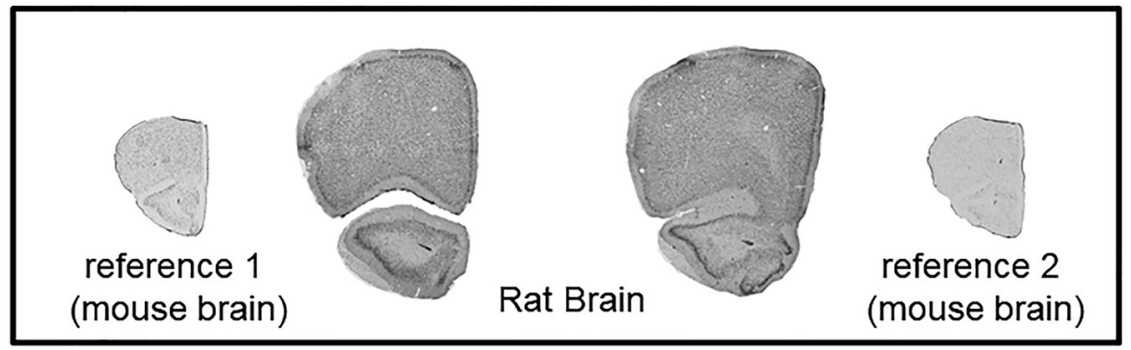

B

Figure 2. Preparation of the brain sections for MALDI-IMS. $\boldsymbol{A}$, Rat brain sections were aligned between adjacent mouse brain sections (references). $\boldsymbol{B}$, Peak intensity of each phospholipid was corrected using the average peak intensity of the same phospholipid of the mouse reference sections.

blood cells were confluent. Blood cells were centrifuged to attach the cells to the surface of the glass slide, fixed with $0.25 \%$ glutaraldehyde for 5 $\mathrm{min}$, and rinsed three times with $150 \mathrm{~mm}$ ammonium acetate buffer, $\mathrm{pH}$ 7.5. Samples were dried and subjected to MALDI-IMS to examine the phospholipid content in the blood cells. Briefly, negative ions from a sample area of $10 \mu \mathrm{m} \times 10 \mu \mathrm{m}$ were obtained in a mass range of $\mathrm{m} / \mathrm{z} 400$ to 1000 . The ratio of the peak intensity of PE $(18: 0 p-22: 6)$ to the total peak intensity of all lipids was compared between the PEL and CL groups for the blood cells collected from the rats of these groups.
Statistical analysis

All data are presented as mean \pm SD. Differences in $\mathrm{BW}$ and locomotor activities in the adult offspring between the 40F, 50F, LPD, or $\mathrm{AL}$ groups were identified using one-way ANOVA followed by Dunnett's multiplecomparison test to examine which treatment leads to the disturbance of the body growth and the behavior in the offspring compared with ad libitum food access. ANCOVA was applied to compare changes in behaviors after liposome injection using the behavioral data at 14 weeks of age as the dependent variable, data at 8 weeks of age as the covariate, and group allocation (CL and PEL groups, or CL, POPEL, and saline groups) as the independent variable. Metabolites in plasma and CSF, gene expression levels, the number of microglia, and phospholipid levels in the brain were compared between the $40 \mathrm{~F}$ and $\mathrm{AL}$ groups by using the unpaired Student's $t$ test. Phospholipid levels in the blood cells were compared among CL, PEL, and saline groups using one-way ANOVA followed by Tukey's HSD test. Differences were considered significant when $p<0.05$, and Cohen's $d$ was calculated to assess the effect size. ANCOVA was performed using JMP version 14.0 software (SAS Institute). The other statistical analyses were performed using SPSS Statistics, version 22.0 software (IBM).

\section{Results}

BW of male rat offspring

No significant difference was observed between the BW of male offspring of $40 \mathrm{~F}$ and that of $\mathrm{AL}, 50 \mathrm{~F}$, or LPD at 9 weeks of age. 
Table 3. Body weight of the offspring ${ }^{a}$

\begin{tabular}{lll}
\hline & Body weight $(\mathrm{g})$ & \\
\cline { 2 - 3 } Group & 9 weeks of age & 12 weeks of age \\
\hline AL & $271.4 \pm 26.5(n=32)$ & $315.5 \pm 26.0(n=18)$ \\
$50 \mathrm{~F}$ & $248.7 \pm 20.6^{*}(n=18)$ & $317.7 \pm 26.2(n=18)$ \\
$40 \mathrm{~F}$ & $267.8 \pm 28.8(n=18)$ & Not measured \\
LPD & $250.4 \pm 17.3^{*}(n=19)$ & $325.7 \pm 18.5(n=19)$ \\
\hline
\end{tabular}

${ }^{a}$ Data are mean $\pm S D$

${ }^{*} p<0.05$ versus AL (one-way ANOVA with Dunnett's test).

However, the BW of the male offspring was significantly lower in the $50 \mathrm{~F}$ and LPD groups ( $p=0.06$ and $p=0.10$, respectively) compared with that of the male offspring in the AL group using Dunnett's test following one-way ANOVA $\left(p=0.002, \eta_{\mathrm{p}}^{2}=\right.$ 0.159 for the main effect) (Table 3 ). The BW of 50F and LPD male offspring became similar to that of AL offspring at 12 weeks of age (Table 3).

\section{Maternal undernutrition during early pregnancy leads to hyperactivity in rat offspring}

To study the effect of nutritional stress during early embryonic stages on postnatal behavior, the open-field test was performed for adult male offspring delivered from dams that underwent food restriction from GD 5.5-10.5. In this study, the behavioral tests performed for offspring at 8 weeks of age revealed that the total distance traveled, the distance traveled in the center area, and the frequency of crossings were significantly increased for males from the 40F group $(p=0.028, p=0.036$, and $p<0.001$, respectively), the 50F group ( $p<0.001, p=0.004$, and $p<0.001$, respectively), and the LPD group $(p=0.026, p=0.030$, and $p=$ 0.016 , respectively) compared with the corresponding findings for males in the AL group, in which dams were fed ad libitum, using Dunnett's test following one-way ANOVA (total distance traveled: $p<0.001, \eta_{\mathrm{p}}^{2}=0.209$; distance traveled in the center: $p=0.003, \eta_{\mathrm{p}}^{2}=0.167$; frequency of crossing: $p<0.001, \eta_{\mathrm{p}}^{2}=$ 0.439 for the main effect; Fig. $1 B-D$ ). There was no significant difference in time spent in the center area among all groups in Condition 1 of the open-field test (Fig. $1 E$ ), but the time spent in the center area was longer for both the $40 \mathrm{~F}(p=0.015)$ and $50 \mathrm{~F}$ $(p=0.037)$ groups compared with the AL group in Condition 2, where the radius of the center area represented $70 \%$ of the open field (Dunnett's test following one-way ANOVA: $p=0.011, \eta_{\mathrm{p}}^{2}=$ 0.135 for the main effect; Fig. $1 F)$. Maternal protein restriction during early pregnancy, in part, contributed to the hyperactivity of the offspring. Increased total distance traveled (LPD: $p<$ 0.001; 50F: $p<0.001)$, the distance traveled in the center area (LPD: $p<0.001$; 50F: $p=0.006$ ), the frequency of crossings (LPD: $p=0.001 ; 50 \mathrm{~F}: p=0.011$ ), and the time spent in the center area in Conditions 1 (LPD: $p=0.020$; 50F: $p=0.015$ ) and 2 (LPD: $p=0.001 ; 50 \mathrm{~F}: p=0.019$ ) were observed, even at 12 weeks of age for offspring from the $50 \mathrm{~F}$ and LPD groups compared with the AL group (one-way ANOVA with Dunnett's test), although this parameter was not examined for the offspring of the $40 \mathrm{~F}$ group (Fig. $1 G-K$ ). However, locomotor activity did not correspond to the BW at 9 and 12 weeks of age (Fig. 1; Table 3). The $p$ value and effect size for the main effect in one-way ANOVA of locomotor activities at 12 weeks of age were as follows: total distance traveled: $p<0.001, \eta_{\mathrm{p}}^{2}=0$. 333; distance traveled in the center: $p<0.001, \eta_{\mathrm{p}}^{2}=0.298$; frequency of crossing: $p=0.001$, $\eta_{\mathrm{p}}^{2}=0.237$; time spent in the center area: $p=0.011, \eta_{\mathrm{p}}^{2}=0.173$ in Condition 1 and $p=0.001, \eta_{\mathrm{p}}^{2}=0.246$ in Condition 2 .

\section{Glyco- and amino-metabolisms are altered in the offspring}

In this study, metabolome profiling of the plasma and CSF was performed for offspring at 9 weeks of age (after behavioral tests) of the $40 \mathrm{~F}$ group, which displayed the most severe behavioral changes among the experimental groups (Fig. 3-1, available at https://doi.org/10.1523/JNEUROSCI.2721-18.2019.f3-1). The concentration of glycerol, which is the source of diacylglycerol in phospholipids, was increased in blood plasma $(p=0.014, d=$ $1.57)$ and $\operatorname{CSF}(p=0.001, d=1.50)$ in the $40 \mathrm{~F}$ group compared with the AL group (Fig. $3 A$ ). In plasma, 1,5-anhydro-D-glucitol $(p=0.038, d=1.61)$ and 2 -aminoethanol $(p=0.014, d=1.21)$ were also increased in the offspring of the 40F group (Fig. $3 A$; Fig. 3-1, available at https://doi.org/10.1523/JNEUROSCI.2721-18. 2019.f3-1). 2-Aminoethanol is converted to O-phosphoethanolamine, and finally transferred to diacylglycerol or 1-O-alkyl2-acyl-sn-glycerol to produce PE (Braverman and Moser, 2012; Vance, 2015).

\section{Microglial cell activation in the PFC is not induced by prenatal undernutrition}

The cell densities of Iba1-positive microglial cells and those of both Iba1- and CD11b-positive activated microglial cells in the PFC were not significantly different between the AL and $40 \mathrm{~F}$ groups (Fig. $3 B-D$ ). Additionally, no change in the ratio of activated glial cells to total microglial cells was observed between the $\mathrm{AL}$ and $40 \mathrm{~F}$ groups (Fig. $3 \mathrm{E}$ ); therefore, microglial cell activation was not enhanced by prenatal undernutrition during the early embryonic period.

\section{Expression of genes related to phospholipid biosynthesis is increased in the PFC of rats exposed to prenatal undernutrition}

To examine the modulation of the phospholipid biosynthetic pathway in the PFC, NAcc, and CPu in offspring (Fig. 4) exposed to maternal undernutrition, gene expression of the enzymes involved in this pathway was examined. In the brain, dihydroxyacetone phosphate (DHAP) is the main precursor of phospholipids. DHAP is synthesized from glucose and serves as a precursor of diacylglycerol, which constitutes the hydrophobic tail of phospholipids, such as PC, PE, and PS (Benjamins et al., 2011). Diacylglycerol may be, in part, formed from glycerol, mediated through glycerol 3-phosphate (Fig. 4) (Jenkins and Hajra, 1976). Further, DHAP serves as a precursor of the ether-phospholipid, plasmalogen (Braverman and Moser, 2012). In the first step of the synthetic pathway of plasmalogen, as well as that for diacyl phospholipids, hexokinase $1(H k 1)$ is one of the key enzymes in the regulation of the carbohydrate metabolic rate, which converts glucose to glucose 6-phosphate (McKenna et al., 2011). The expression of $H k 1$ was enhanced in 40F offspring ( $p=0.016, d=$ $1.68)$, although phosphofructokinase $(P f k 1)$, the rate-limiting enzyme in glycolysis (McKenna et al., 2011), was not different in the PFC between 40F and control offspring (Fig. 5A, B; Fig. 5-1, available at https://doi.org/10.1523/JNEUROSCI.2721-18.2019. f5-1). Regarding the synthesis of the hydrophobic tail of phospholipids, the gene expression of the following enzymes related to diacylglycerol and CDP-diacylglycerol synthesis was elevated in the PFC of the offspring of the 40F group: glyceronephosphate O-acyltransferase (Gnpat) $(p=0.001, d=2.32)$, glycerol kinase ( $p=0.016, d=1.60)$, glycerol-3-phosphate transferase (Gpat) 1 , 3 , and $4(p=0.027, p=0.032$, and $p=0.030$, and $d=1.46, d=$ 1.50 , and $d=1.57$, respectively), and phosphatidate cytidylyltransferase $2(p=0.045, d=1.25)$. Regarding the synthesis of the hydrophilic head of phospholipids, the gene expression of etha- 
A

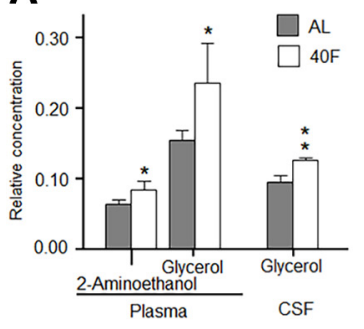

B

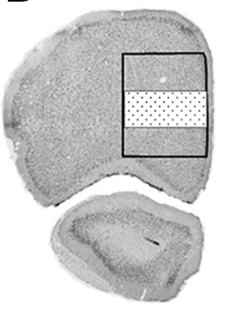

C

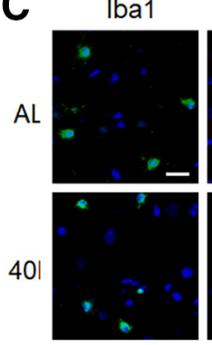

CD11b

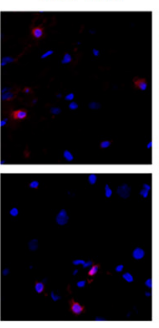

merge

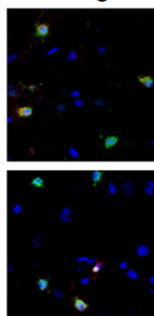

D

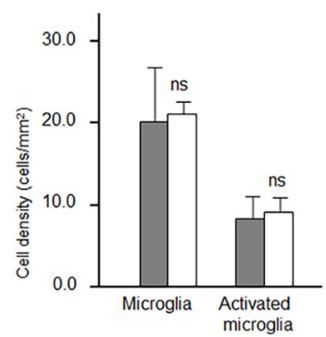

E

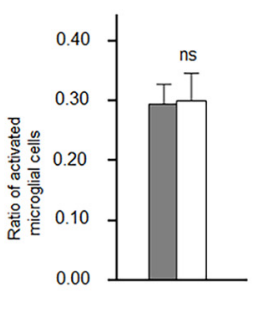

Figure 3. Metabolome profiling of the plasma and CSF, and the observation of microglia in the PFC. A, 2-Aminoethanol and glycerol increased in the rat offspring exposed to prenatal undernutrition (plasma: $n=7$ in AL and $n=6$ in 40F; (SF: $n=7$ in AL and $n=5$ in 40F) (see also Figure 3-1, available at https://doi.org/10.1523/JNEUROSCI.2721-18.2019.f3-1). B, The dotted area, which was the middle third of the box area, was examined. C, Microglial cell activation in the PFC Iba1-positive microglia (green), CD11b-positive cells (red), and activated microglia (yellow) are shown in the PFC of the offspring of the AL and 40F groups. Scale bar, $20 \mu \mathrm{m}$. D, The densities of microglia and activated microglia were not increased in the PFC of $40 \mathrm{~F}(n=8)$ offspring compared with AL $(n=8)$ offspring. $E$, The ratio of the number of activated microglia to the total number of microglia was not altered by prenatal undernutrition. Student's $s$ test: ${ }^{*} p<0.05$; ${ }^{* *} p<0.01$. ns, not significant.

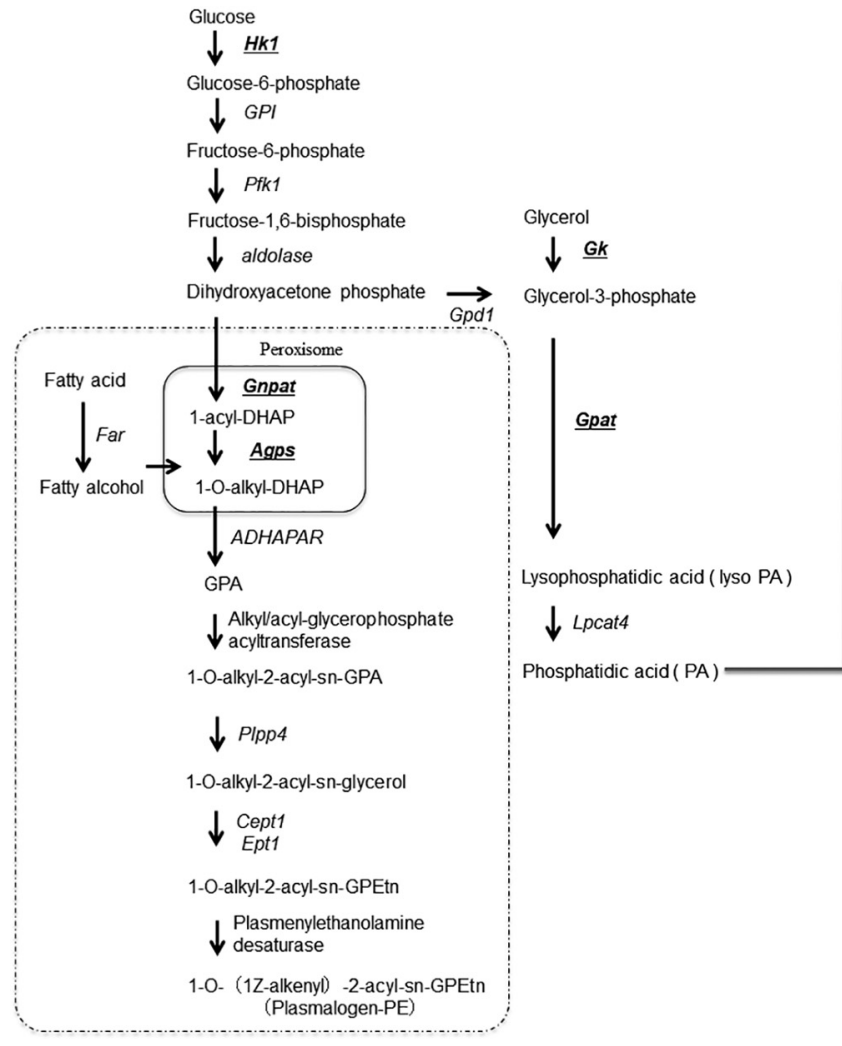

Synthetic pathway of plasmalogens

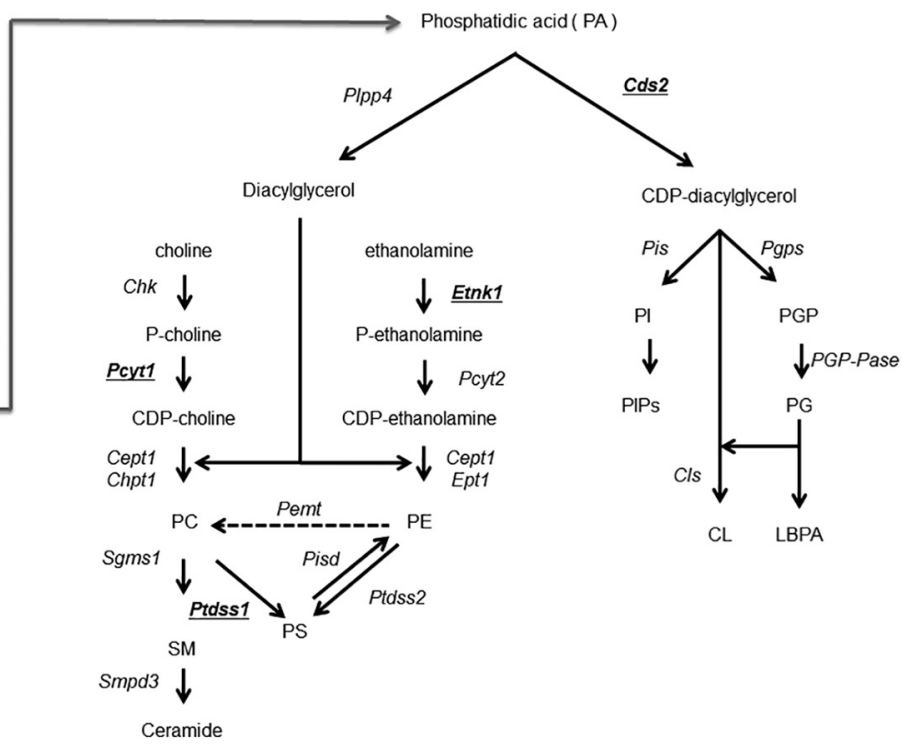

Synthetic pathway of diacyl phospholipids

Figure 4. Biosynthetic pathway of plasmalogens and diacyl phospholipids. The enzymes related to phospholipid synthesis, with intermediates, are shown. The genes indicated by underlined bold italic characters were activated. Abbreviations of the genes are noted as follows: Hk, Hexokinase; GPI, glucose-6-phosphate isomerase; Pfk, phosphofructokinase; gructose-bisphosphate aldolase; Gpd, glycerol-3-phosphate dehydrogenase; Far, fatty acyl-CoA reductase; Gk, glycerol kinase; Gnpat, glyceronephosphate 0-acyltransferase; Agps, alkylglycerone phosphate synthase; ADHAPAR, alkyl/acyl-glycerophosphate acyltransferase; Plpp, phosphatidic acid phosphatase; Cept, choline/ethanolamine phosphotransferase; Ept, ethanolamine phosphotransferase; Chpt, choline phosphotransferase; Gpat, glycerol-3-phosphate acyltransferase; Lpcat, LysoPA-acyltransferase; Chk, choline kinase; Etnk, ethanolamine kinase; Pemt, phosphoethanolamine N-methyltransferase; Pcyt, phosphate cytidyltransferase; Pisd, phosphatidylserine decarboxylase; Ptdss, phosphatidylserine synthase; CDS, CDP-diacylglycerol synthase; Sgms, sphingomyelin synthase; Smpd, sphingomyelin phosphodiesterase; Pis, phosphatidylinositol synthase; Pgps, phosphatidylglycerophosphate synthase; Cls, cardiolipin synthase; CL, cardiolipin; GPA, glycerophosphatidic acid; LBPA, Lysobisphosphatidic acid; $P G$, phosphatidylglycerol; SM, sphingomyelin.

nolamine kinase for PE $(p=0.033, d=1.37)$, phosphate cytidylyltransferase 1 , choline, $\alpha$ (Pcyt1a) $(p=0.013, d=1.68)$ and phosphate cytidylyltransferase 1 , choline, and $\beta$ (Pcyt1b) $(p=$ $0.010, d=1.73$ ) for PC; and phosphatidylserine synthase 1 $(P t d s s 1)$ for PS $(p=0.007, d=1.86)$ was elevated in the PFC of the offspring of the $40 \mathrm{~F}$ group (Fig. $5 \mathrm{~B}$ ). In contrast, plasmalogen is synthesized from ethanolamine or choline and 1-O-alkyl-2- acyl-sn-glycerol, which is produced from fatty alcohol and 1-Oalkyl-DHAP (Braverman and Moser, 2012). In this pathway, 1-O-alkyl-DHAP is generated from DHAP by GNPAT and alkylglycerone phosphate synthase (AGPS) (Braverman and Moser, 2012). The genes, AGPS ( $p=0.013, d=1.71)$ and GNPAT of these enzymes were found to be activated, whereas the fatty acyl-CoA reductase 1 (Far1) gene expression, which is a potential rate-limiting 
A
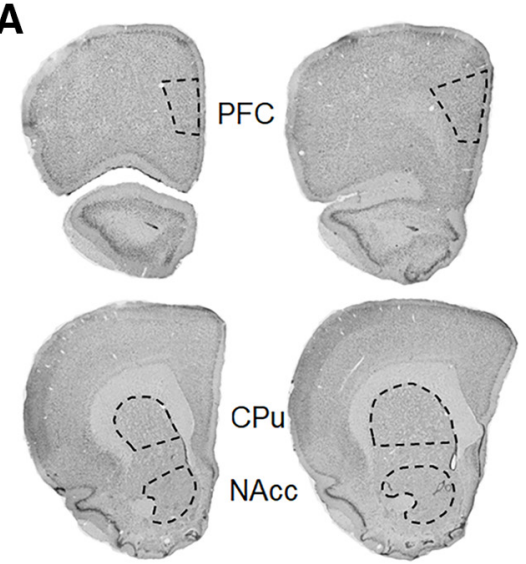

C
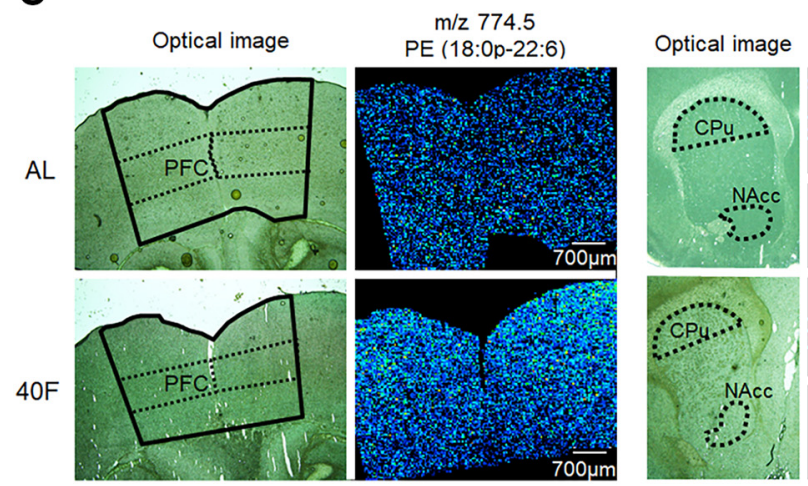

E

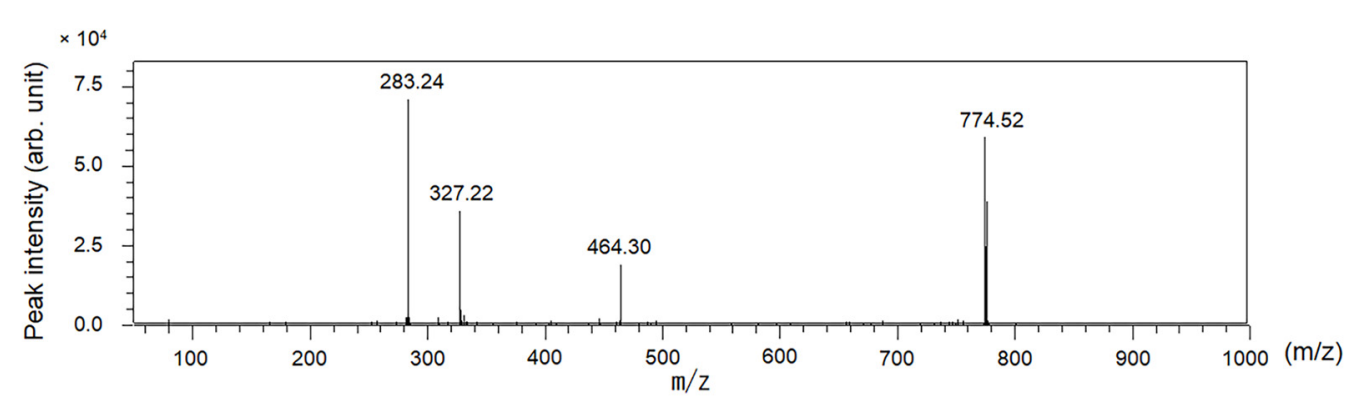

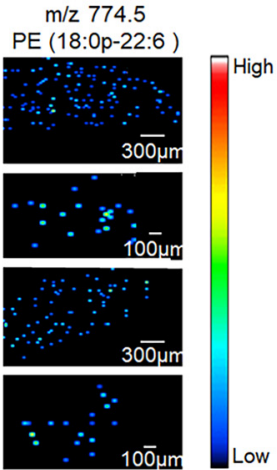

B
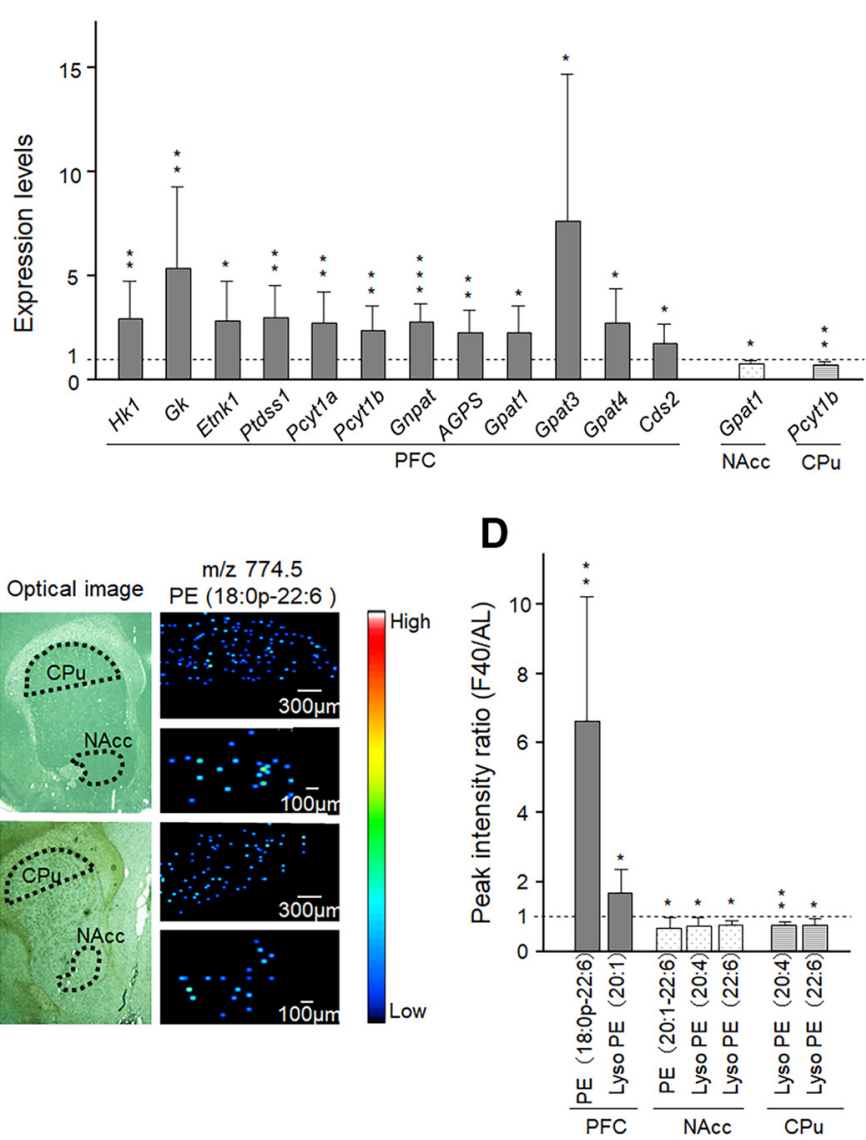

Figure 5. Gene expression of the enzymes related to phospholipid synthesis and phospholipid composition of the rat brain. $\boldsymbol{A}$, The area examined by gene expression analysis. $\boldsymbol{B}$, The ratios of the expression levels of the enzymes in the synthetic pathway of plasmalogens and diacyl phospholipids in the PFC, NAcc, and CPu for the 40F group offspring $(n=8)$ were compared with those for the AL group offspring $(n=8)$ (see Figure 5-1, available at https://doi.org/10.1523/JNEUROSCI.2721-18.2019.f5-1). C, The area examined by MALDI-IMS. The dotted areas were examined in the PFC, NAcc, and CPu. In a section of the PFC, the dotted area is the middle third of the box area indicated by the solid line. Signal intensity was indicated by color. $D$, The ratios of peak intensities of phospholipids of $40 \mathrm{~F}$ offspring $(n=7)$ to those of AL offspring $(n=7)$ are shown (see Figure 5-2, available at https://doi.org/10.1523/JNEUROSCI.2721-18.2019.f5-2).E, The peaks of product ions by collision-induced dissociation of $\mathrm{m} / \mathrm{z} 774.5$ in MALDI tandem mass spectrometry. Student's $t$ test: ${ }^{*} p<0.05 ;{ }^{* *} p<0.01 ;{ }^{* * *} p=0.001$.

enzyme (Honsho and Fujiki, 2017), was not altered in the offspring of the $40 \mathrm{~F}$ group compared with that of the offspring of the AL group (Fig. 5B; Fig. 5-1, available at https://doi.org/10.1523/ JNEUROSCI.2721-18.2019.f5-1). In contrast to the PFC, Gpat1 $(p=0.036, d=1.39)$ and Pcyt $1 b(p=0.008, d=1.65)$ expression was lower in the offspring of the $40 \mathrm{~F}$ group than in the offspring of the AL group in the NAcc and $\mathrm{CPu}$, respectively, although the expression of the other genes, which showed altered expression in the PFC, was not changed in the NAcc and $\mathrm{CPu}$ (Fig. 5A,B; Fig. 5-1, available at https://doi.org/10.1523/ JNEUROSCI.2721-18.2019.f5-1). Furthermore, between the offspring of the $\mathrm{AL}$ group and the $40 \mathrm{~F}$ group, in the $\mathrm{PFC}$, no significant difference was observed in the expression of the calcium-independent phospholipase A2 (iPla2) gene, which catalyzes phospholipids (Yeagle, 2016), or that of sphingomyelin synthase 1 (Sgms1) and sphingomyelin phosphodiesterase 3 (Smpd3), which convert PC into sphingomyelin and ceramide (Vance, 2015), respectively (Fig. 5-1, available at https://doi.org/ 10.1523/JNEUROSCI.2721-18.2019.f5-1). As described above, our results indicated that the genes of enzymes related to plasmalogen, as well as diacyl phospholipids, were activated in the PFC of offspring exposed to prenatal undernourishment. Alternatively, gene expression was not affected with respect to aquaporin 9 (Aqp9; a channel permeable to glycerol and water) (Badaut and 
Table 4. Change in amounts of DHA- or AA-containing phospholipids in PFC, NAcc, and CPu by undernutrition ${ }^{a}$

\begin{tabular}{|c|c|c|c|c|c|c|c|}
\hline \multirow{2}{*}{ Phospholipid } & \multirow[b]{2}{*}{ sn1_sn2 } & \multicolumn{2}{|l|}{ PFC } & \multicolumn{2}{|l|}{ NACC } & \multicolumn{2}{|l|}{$\mathrm{CPu}$} \\
\hline & & $\mathrm{AL}$ & $40 \mathrm{~F}$ & $\mathrm{AL}$ & $40 \mathrm{~F}$ & $\mathrm{AL}$ & $40 \mathrm{~F}$ \\
\hline Lyso PE_AA & 20:4 (acyl) & $3.86 \pm 1.20$ & $6.30 \pm 2.70$ & $3.60 \pm 0.67$ & $2.60 \pm 0.77^{*}$ & $3.76 \pm 0.57$ & $2.75 \pm 0.39^{*}$ \\
\hline Lyso PE_DHA & 22:6 (acyl) & $2.54 \pm 0.84$ & $3.67 \pm 1.17$ & $2.23 \pm 0.46$ & $1.70 \pm 0.26^{*}$ & $2.48 \pm 0.33$ & $1.92 \pm 0.38^{*}$ \\
\hline \multirow[t]{2}{*}{ PE_AA_Pls } & 16:0p-20:4 (alkenyl_acyl) & $2.83 \pm 0.95$ & $3.97 \pm 1.70$ & $4.52 \pm 1.46$ & $4.52 \pm 0.86$ & $3.27 \pm 0.74$ & $3.46 \pm 0.76$ \\
\hline & 18:1p-20:4 (alkenyl_acyl) & $2.07 \pm 0.72$ & $2.48 \pm 1.03$ & $2.15 \pm 0.41$ & $2.02 \pm 0.43$ & $1.94 \pm 0.27$ & $1.90 \pm 0.42$ \\
\hline \multirow[t]{5}{*}{ PE_AA_acyl } & 18:1-20:4 (alkyl_acyl) & $2.71 \pm 0.86$ & $3.39 \pm 1.31$ & $3.65 \pm 0.82$ & $3.38 \pm 0.63$ & $2.89 \pm 0.50$ & $3.00 \pm 0.62$ \\
\hline & 18:0 -20:4 (alkyl_acyl) & $2.95 \pm 0.73$ & $4.29 \pm 1.87$ & $3.83 \pm 0.95$ & $3.21 \pm 0.72$ & $3.14 \pm 0.76$ & $2.97 \pm 0.59$ \\
\hline & $18: 2-20: 4$ (diacyl) & $1.37 \pm 0.47$ & $1.67 \pm 0.67$ & $1.21 \pm 0.22$ & $1.19 \pm 0.26$ & $1.18 \pm 0.17$ & $1.19 \pm 0.27$ \\
\hline & 18:1-20:4 (diacyl) & $1.67 \pm 0.54$ & $2.10 \pm 0.88$ & $1.64 \pm 0.31$ & $1.45 \pm 0.26$ & $1.67 \pm 0.20$ & $1.49 \pm 0.27$ \\
\hline & 18:0-20:4 (diacyl) & $1.84 \pm 0.56$ & $2.35 \pm 0.96$ & $2.38 \pm 0.32$ & $2.11 \pm 0.42$ & $1.74 \pm 0.19$ & $1.72 \pm 0.40$ \\
\hline \multirow[t]{3}{*}{ PE_DHA_Pls } & 18:0p-22:6 (alkenyl_acyl) & $1.41 \pm 0.43$ & $9.39 \pm 4.62^{*}$ & $1.74 \pm 0.32$ & $1.67 \pm 0.30$ & $1.79 \pm 0.28$ & $1.75 \pm 0.32$ \\
\hline & 18:1p-22:6 (alkenyl_acyl) & $1.30 \pm 0.36$ & $1.63 \pm 0.65$ & $1.28 \pm 0.24$ & $1.16 \pm 0.20$ & $1.34 \pm 0.26$ & $1.26 \pm 0.19$ \\
\hline & 20:1p-22:6 (alkenyl_acyl) & $3.11 \pm 1.38$ & $3.46 \pm 0.85$ & $2.09 \pm 0.69$ & $1.77 \pm 0.82$ & $2.48 \pm 1.14$ & $2.13 \pm 0.49$ \\
\hline \multirow[t]{9}{*}{ PE_DHA_acyl } & $16: 1-22: 6$ (diacyl) & $1.36 \pm 0.38$ & $1.64 \pm 0.84$ & $1.80 \pm 0.41$ & $1.55 \pm 0.26$ & $1.86 \pm 0.24$ & $1.66 \pm 0.20$ \\
\hline & 18:0 -22:6 (alkyl_acyl) & $1.67 \pm 0.46$ & $2.19 \pm 0.92$ & $1.94 \pm 0.45$ & $1.70 \pm 0.34$ & $1.93 \pm 0.35$ & $1.93 \pm 0.41$ \\
\hline & $18: 2-22: 6$ (diacyl) & $1.75 \pm 0.66$ & $2.27 \pm 1.09$ & $1.56 \pm 0.29$ & $1.37 \pm 0.31$ & $1.93 \pm 0.32$ & $1.73 \pm 0.42$ \\
\hline & 18:0-22:6 (diacyl) & $1.31 \pm 0.42$ & $1.67 \pm 0.63$ & $1.46 \pm 0.28$ & $1.46 \pm 0.31$ & $1.44 \pm 0.22$ & $1.48 \pm 0.31$ \\
\hline & 20:1-22:6 (diacyl) & $3.00 \pm 1.17$ & $3.65 \pm 1.96$ & $2.72 \pm 0.71$ & $1.76 \pm 0.77^{*}$ & $3.08 \pm 0.91$ & $2.69 \pm 1.20$ \\
\hline & 20:0-22:6 (diacyl) & $2.16 \pm 0.85$ & $3.42 \pm 1.71$ & $3.28 \pm 1.08$ & $2.58 \pm 1.73$ & $4.47 \pm 1.45$ & $3.74 \pm 2.08$ \\
\hline & $22: 6-22: 6$ (diacyl) & $1.86 \pm 0.72$ & $2.35 \pm 0.98$ & $1.87 \pm 0.35$ & $1.76 \pm 0.40$ & $1.74 \pm 0.29$ & $1.73 \pm 0.39$ \\
\hline & $22: 6-24: 6$ (diacyl) & $2.74 \pm 2.55$ & $2.90 \pm 0.55$ & $1.41 \pm 0.40$ & $1.05 \pm 0.28$ & $3.52 \pm 1.30$ & $3.23 \pm 0.81$ \\
\hline & $24: 4-22: 6$ (diacyl) & $2.97 \pm 1.20$ & $3.55 \pm 1.41$ & $1.74 \pm 0.58$ & $2.01 \pm 1.46$ & $2.24 \pm 0.80$ & $2.49 \pm 1.23$ \\
\hline PS_AA_acyl & 18:1-20:4 (diacyl) & $2.00 \pm 0.63$ & $2.94 \pm 1.50$ & $3.50 \pm 1.28$ & $3.16 \pm 0.76$ & $6.01 \pm 1.91$ & $6.38 \pm 1.87$ \\
\hline \multirow[t]{4}{*}{ PS_DHA_acyl } & $16: 0-22: 6$ (diacyl) & $1.46 \pm 0.47$ & $2.39 \pm 1.13$ & $3.91 \pm 0.94$ & $3.35 \pm 0.68$ & $7.26 \pm 1.62$ & $7.21 \pm 1.56$ \\
\hline & 18:1-22:6 (diacyl) & $2.64 \pm 0.97$ & $3.12 \pm 1.57$ & $1.73 \pm 0.63$ & $1.63 \pm 0.43$ & $2.14 \pm 0.46$ & $1.88 \pm 0.38$ \\
\hline & 20:0-22:6 (diacyl) & $2.11 \pm 0.67$ & $4.29 \pm 2.46$ & $2.12 \pm 0.92$ & $1.89 \pm 0.54$ & $2.72 \pm 1.02$ & $2.47 \pm 0.50$ \\
\hline & $22: 4-22: 6$ (diacyl) & $2.10 \pm 1.19$ & $3.56 \pm 2.64$ & $1.56 \pm 0.22$ & $1.56 \pm 0.44$ & $1.91 \pm 0.29$ & $1.77 \pm 0.45$ \\
\hline PS_AA_DHA & 20:4-22:6 (diacyl) & $2.09 \pm 0.77$ & $2.52 \pm 1.08$ & $2.02 \pm 0.57$ & $1.82 \pm 0.44$ & $2.01 \pm 0.69$ & $2.16 \pm 0.77$ \\
\hline Lyso PI_AA & 20:4 (acyl) & $3.40 \pm 1.67$ & $4.53 \pm 2.83$ & $4.39 \pm 1.03$ & $4.06 \pm 2.43$ & $4.22 \pm 0.64$ & $3.99 \pm 2.32$ \\
\hline \multirow[t]{5}{*}{ PI_AA_acyl } & 16:1-20:4 (diacyl) & $5.79 \pm 8.33$ & $4.14 \pm 2.30$ & $1.92 \pm 0.39$ & $1.87 \pm 0.50$ & $2.44 \pm 0.66$ & $2.21 \pm 0.22$ \\
\hline & 16:0-20:4 (diacyl) & $2.07 \pm 0.84$ & $2.38 \pm 1.07$ & $4.77 \pm 7.19$ & $1.44 \pm 0.49$ & $4.67 \pm 6.92$ & $1.52 \pm 0.50$ \\
\hline & 18:0 -20:4 (alkyl_acyl) & $2.83 \pm 1.19$ & $2.67 \pm 1.19$ & $1.98 \pm 0.56$ & $1.82 \pm 0.28$ & $2.27 \pm 0.53$ & $2.11 \pm 0.30$ \\
\hline & $18: 1-20: 4$ (diacyl) & $1.89 \pm 0.77$ & $2.23 \pm 1.01$ & $1.70 \pm 0.36$ & $1.42 \pm 0.49$ & $1.59 \pm 0.32$ & $1.43 \pm 0.42$ \\
\hline & 18:0-20:4 (diacyl) & $2.28 \pm 0.83$ & $2.79 \pm 1.19$ & $2.71 \pm 0.77$ & $2.66 \pm 0.78$ & $2.80 \pm 0.39$ & $2.69 \pm 0.75$ \\
\hline PI_DHA_acyl & 18:0-22:6 (diacyl) & $2.22 \pm 0.87$ & $2.82 \pm 1.80$ & $1.17 \pm 0.33$ & $1.17 \pm 0.35$ & $1.64 \pm 0.33$ & $1.34 \pm 0.56$ \\
\hline
\end{tabular}

${ }^{a}$ Data are mean \pm SD.

${ }^{*} p<0.05$.

Regli, 2004) or major facilitator superfamily domain containing 2A (Mfsd2a; the major transporter for docosahexaenoic acid [DHA]) (Nguyen et al., 2014). Additionally, the expression of apolipoprotein E, which is involved in lipid transport (Liao et al., 2017), was not altered by prenatal undernutrition (Fig. 5-1, available at https://doi.org/10.1523/JNEUROSCI.2721-18.2019.f5-1).

\section{Phospholipid composition is altered in the cerebrum by maternal undernutrition}

The peak intensity of $\mathrm{m} / \mathrm{z} 774.5 \mathrm{PE}$ in the PFC of the offspring of the $40 \mathrm{~F}$ group was 6.6-fold higher than that of the offspring of the AL group $(p=0.012, d=2.33$; Fig. $5 C, D)$. Collision-induced dissociation of $\mathrm{m} / \mathrm{z} 774.5$ yielded product ions at $\mathrm{m} / \mathrm{z} 283.2$, 327.2, and 464.3; therefore, $\mathrm{m} / \mathrm{z} 774.5$ was identified as PE (18: 0p-22:6) (Fig. 5E). Additionally, lyso-PE (20:1) in the PFC of $40 \mathrm{~F}$ offspring was 1.7 -fold that of the AL offspring $(p=0.037, d=$ 1.36); however, no significant difference was observed between groups for the other phospholipids examined (Fig. 5-2, available at https://doi.org/10.1523/JNEUROSCI.2721-18.2019.f5-2). In contrast, the peak intensities of PE (20:1-22:6), lyso-PE (20:4), and lyso-PE (22:6) in the NAcc $(p=0.045, p=0.034$, and $p=$ 0.031 , and $d=1.30, d=1.39$, and $d=1.42$, respectively), and of lyso-PE (20:4) and lyso-PE (22:6) in the $\mathrm{CPu}(p=0.004$ and $p=$ 0.019 , and $d=2.07$ and $d=1.57$, respectively) were attenuated in the offspring of the $40 \mathrm{~F}$ group compared with the offspring of the AL group. However, no phospholipid showed enhanced peak intensity in the NAcc and CPu of the offspring of the $40 \mathrm{~F}$ group (Fig. 5D; Fig. 5-2, available at https://doi.org/10.1523/ JNEUROSCI.2721-18.2019.f5-2). On the other hand, only PE (18:0p-22:6) in the PFC varied in amount among PlsEtn examined in this study by prenatal undernutrition (Fig. 5D; Table 4). From the perspective of the tail forms of the phospholipids, $\mathrm{PE}$ (18:0p-22:6) in the PFC exclusively increased in the offspring of the $40 \mathrm{~F}$ group among DHA (22:6)-containing phospholipids, although some phospholipids containing DHA or arachidonic acid (AA) (20:4) decreased in the NAcc and CPu (Fig. 5D; Table 4).

\section{PlsEtn affects the behavior of adult rats}

To examine the effect of PE (18:0p-22:6) on rat behavior, rats were subjected to the open-field test and elevated plus maze test after intravenous injection of PEL or CL (Fig. 6A). The agerelated decline in the frequency of crossing, as well as the difference in the time spent in the center area (Condition 2) was reduced in rats in the PEL group compared with rats in the CL group (Fig. 6B). However, no significant difference was observed in the results of the elevated plus maze test between the PEL and CL groups (Fig. 6D). At the same time, no significant difference was observed in the effect of POPEL, CL, and saline on rat behavior (Fig. 6C,E). Four days after the second injection of PEL (Fig. $6 A)$, the amount of PE (18:0p-22:6) in the PFC $(p=0.019, d=$ 3.02 ), but not that in the NAcc or $\mathrm{CPu}$, was still greater in the PEL group than in the CL group, while no significant difference was 
A
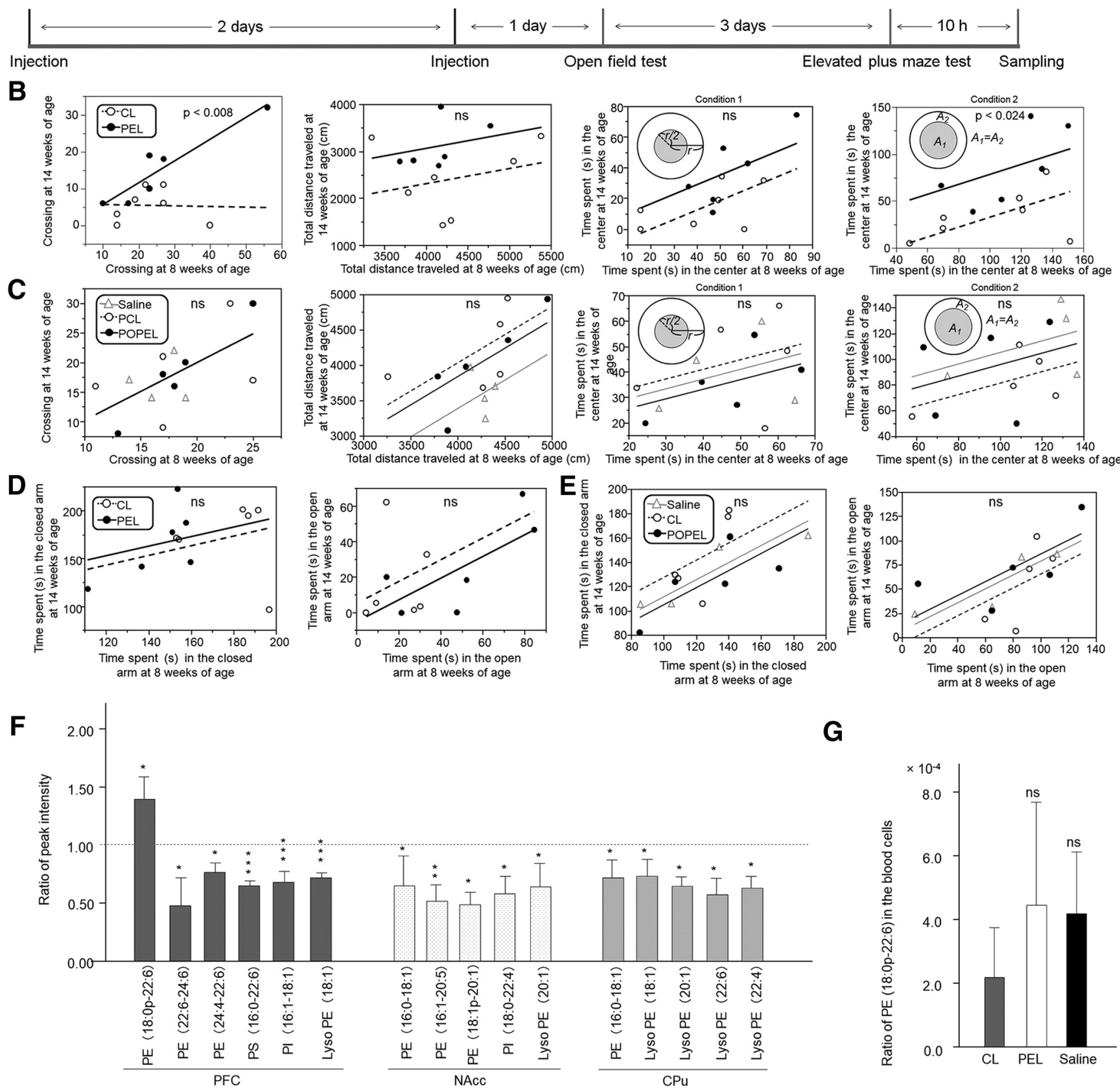

Figure 6. Behavioral tests and phospholipid composition in the brain of the rats injected with PE. $A$, The experimental schedule of the liposome injection at 14 weeks of age. Changes in the behavior of rats by using the open-field test after (B) PE (18:0p-22:6) (PEL: $n=6 ; \mathrm{CL}: n=7)$, or (C) POPE injection (POPEL: $n=5 ;(\mathrm{CL}: n=5 ;$ saline: $n=4)$ were examined by ANCOVA. Similarly, behavioral changes evaluated according to the elevated plus maze test after (D) PE (18:0p-22:6) (PEL: $n=6 ;(\mathrm{CL}: n=6)$, or (E) POPE injection (POPEL: $n=5$; CL: $n=5$; saline: $n=4)$ were examined. $\boldsymbol{F}$, The ratios of peak intensities of phospholipids in the brains of PEL-injected rats $(n=4)$ significantly increased compared with those of CL-injected rats $(n=4)$ using Student's $t$ test (see also Figure 6-1, available at https://doi.org/10.1523/JNEUROSCI.2721-18.2019.f6-1). The increased ratio of peak intensity of PE (18:0p-22:6) in the PFC of PEL-injected rats was verified in an additional experiment (see Figure 6-2, available at https://doi.org/10.1523/JNEUROSCI.2721-18.2019.f6-2). G, The ratio of PE (18:0p-22:6) to total lipids in the blood cells was compared among CL PEL and saline groups using one-way ANOVA with Tukey's HSD test. ${ }^{*} p<0.05,{ }^{* *} p<0.01,{ }^{* * *} p<0.001$. ns, not significant.

observed in the amount of the other PlsEtn between these groups, as determined by MALDI-IMS (Fig. 6F; Fig. 6-1, available at https://doi.org/10.1523/JNEUROSCI.2721-18.2019.f6-1). The increased amount of PE (18:0p-22:6) in the PFC of the PEL group was verified in another PE injection experiment (Fig. 6-2, available at https://doi.org/10.1523/JNEUROSCI.2721-18.2019.f6-2). Meanwhile, the amounts of PE $(22: 6-24: 6)(p=0.019, d=$ 3.02), PE (24:4-22:6) ( $p=0.013, d=3.36), \operatorname{PS}(16: 0-22: 6)(p<$ $0.001, d=9.02)$, PI $(16: 1-18: 1)(p=0.004, d=4.56)$, and
lyso-PE (18:1) ( $p<0.001, d=8.58)$, all of which are acyl phospholipids, were lower in the PFC in the PEL group than in the CL group (Fig. $6 F$ ). The amounts of PE (16:0-18:1) (NAcc; $p=$ 0.041, $d=2.08$, CPu: $p=0.016, d=2.68)$ and lyso-PE $(20: 1)$ (NAcc; $p=0.012, d=2.91, \mathrm{CPu}: p=0.033, d=2.64$ ) were lower in the NAcc and CPu for the PEL group than in the CL group. The amounts of PE (16:1-20:5), PI (18:0-22:4), and plasmalogen PE $(18: 1 \mathrm{p}-20: 1)$ were also decreased in the NAcc of the PEL group $(p=0.008, p=0.032$, and $p=0.023$, and $d=3.18, d=0.85$, and 
Table 5. Change in amounts of DHA- or AA-containing phospholipids in PFC, NAcc, and CPu of the rats injected with liposomes ${ }^{a}$

\begin{tabular}{|c|c|c|c|c|c|c|c|}
\hline \multirow{2}{*}{ Phospholipid } & \multirow[b]{2}{*}{ sn1_sn2 } & \multicolumn{2}{|l|}{ PFC } & \multicolumn{2}{|l|}{ NACC } & \multicolumn{2}{|l|}{$\mathrm{CPu}$} \\
\hline & & PEL & $\mathrm{CL}$ & PEL & $\mathrm{CL}$ & PEL & $\mathrm{CL}$ \\
\hline Lyso PE_AA & 20:4 (acyl) & $1.94 \pm 0.21$ & $2.17 \pm 0.24$ & $1.44 \pm 0.79$ & $2.15 \pm 0.88$ & $1.31 \pm 0.15$ & $1.87 \pm 0.58$ \\
\hline Lyso PE_DHA & 22:6 (acyl) & $1.29 \pm 0.23$ & $1.55 \pm 0.09$ & $1.00 \pm 0.45$ & $1.24 \pm 0.28$ & $0.88 \pm 0.19^{*}$ & $1.55 \pm 0.32$ \\
\hline \multirow[t]{2}{*}{ PE_AA_Pls } & 16:0p-20:4 (alkenyl_acyl) & $4.29 \pm 0.71$ & $3.66 \pm 0.59$ & $3.02 \pm 1.03$ & $3.36 \pm 0.50$ & $2.19 \pm 0.43$ & $2.58 \pm 0.62$ \\
\hline & 18:1p-20:4 (alkenyl_acyl) & $2.25 \pm 0.41$ & $2.07 \pm 0.32$ & $1.13 \pm 0.36$ & $1.36 \pm 0.14$ & $1.06 \pm 0.24$ & $1.24 \pm 0.20$ \\
\hline \multirow[t]{5}{*}{ PE_AA_acyl } & 18:1-20:4 (alkyl_acyl) & $3.22 \pm 0.54$ & $2.70 \pm 0.58$ & $2.26 \pm 0.73$ & $2.48 \pm 0.19$ & $1.88 \pm 0.42$ & $2.14 \pm 0.36$ \\
\hline & 18:0-20:4 (alkyl_acyl) & $3.11 \pm 0.58$ & $2.28 \pm 0.27$ & $2.11 \pm 0.65$ & $2.56 \pm 0.32$ & $1.82 \pm 0.40$ & $2.30 \pm 0.29$ \\
\hline & $18: 2-20: 4$ (diacyl) & $1.87 \pm 0.28$ & $1.50 \pm 0.24$ & $0.65 \pm 0.18$ & $0.73 \pm 0.05$ & $0.68 \pm 0.13$ & $0.78 \pm 0.10$ \\
\hline & 18:1-20:4 (diacyl) & $1.69 \pm 0.39$ & $1.43 \pm 0.28$ & $1.02 \pm 0.24$ & $1.12 \pm 0.17$ & $1.06 \pm 0.20$ & $1.19 \pm 0.24$ \\
\hline & 18:0-20:4 (diacyl) & $2.60 \pm 0.56$ & $1.97 \pm 0.60$ & $1.30 \pm 0.35$ & $1.50 \pm 0.12$ & $1.07 \pm 0.20$ & $1.22 \pm 0.16$ \\
\hline \multirow[t]{3}{*}{ PE_DHA_Pls } & 18:0p-22:6 (alkenyl_acyl) & $2.62 \pm 0.31^{*}$ & $1.88 \pm 0.08$ & $1.06 \pm 0.30$ & $1.18 \pm 0.05$ & $1.08 \pm 0.18$ & $1.18 \pm 0.13$ \\
\hline & 18:1p-22:6 (alkenyl_acyl) & $2.13 \pm 0.31$ & $1.99 \pm 0.16$ & $0.73 \pm 0.23$ & $0.88 \pm 0.11$ & $0.84 \pm 0.15$ & $1.00 \pm 0.15$ \\
\hline & 20:1p-22:6 (alkenyl_acyl) & $2.99 \pm 1.29$ & $2.39 \pm 0.22$ & $1.00 \pm 0.46$ & $1.04 \pm 0.26$ & $1.33 \pm 0.30$ & $1.92 \pm 0.64$ \\
\hline \multirow[t]{9}{*}{ PE_DHA_acyl } & 16:1-22:6 (diacyl) & $1.10 \pm 0.31$ & $1.03 \pm 0.19$ & $0.97 \pm 0.27$ & $1.20 \pm 0.28$ & $1.26 \pm 0.26$ & $1.32 \pm 0.27$ \\
\hline & 18:0 -22:6 (alkyl_acyl) & $2.52 \pm 0.38$ & $1.87 \pm 0.35$ & $0.99 \pm 0.31$ & $1.23 \pm 0.08$ & $1.17 \pm 0.22$ & $1.33 \pm 0.15$ \\
\hline & $18: 2-22: 6$ (diacyl) & $1.29 \pm 0.24$ & $1.27 \pm 0.20$ & $0.86 \pm 0.17$ & $1.08 \pm 0.22$ & $1.04 \pm 0.34$ & $1.31 \pm 0.31$ \\
\hline & 18:0-22:6 (diacyl) & $1.63 \pm 0.32$ & $1.28 \pm 0.33$ & $0.82 \pm 0.20$ & $0.92 \pm 0.10$ & $0.84 \pm 0.14$ & $0.93 \pm 0.11$ \\
\hline & 20:1-22:6 (diacyl) & $1.94 \pm 0.36$ & $2.05 \pm 0.46$ & $0.88 \pm 0.31$ & $1.08 \pm 0.24$ & $0.92 \pm 0.44$ & $1.19 \pm 0.39$ \\
\hline & 20:0-22:6 (diacyl) & $1.85 \pm 0.46$ & $2.12 \pm 0.21$ & $1.90 \pm 1.23$ & $3.23 \pm 0.69$ & $2.68 \pm 0.40$ & $3.44 \pm 0.58$ \\
\hline & 22:6-22:6 (diacyl) & $1.91 \pm 0.41$ & $1.71 \pm 0.40$ & $1.05 \pm 0.32$ & $1.31 \pm 0.13$ & $1.02 \pm 0.21$ & $1.20 \pm 0.21$ \\
\hline & 22:6-24:6 (diacyl) & $3.74 \pm 1.60^{*}$ & $7.77 \pm 0.78$ & $0.61 \pm 0.28$ & $0.93 \pm 0.59$ & $1.68 \pm 0.44$ & $2.23 \pm 0.50$ \\
\hline & 24:4-22:6 (diacyl) & $2.28 \pm 0.22^{*}$ & $2.98 \pm 0.19$ & $1.08 \pm 0.75$ & $1.54 \pm 0.20$ & $0.98 \pm 0.22$ & $1.43 \pm 0.55$ \\
\hline PS_AA_acyl & 18:1-20:4 (diacyl) & $1.04 \pm 0.14$ & $1.34 \pm 0.14$ & $1.73 \pm 0.67$ & $2.03 \pm 0.25$ & $2.77 \pm 0.69$ & $3.32 \pm 0.49$ \\
\hline \multirow[t]{4}{*}{ PS_DHA_acyl } & $16: 0-22: 6$ (diacyl) & $0.60 \pm 0.03^{*}$ & $0.91 \pm 0.04$ & $1.60 \pm 0.62$ & $2.41 \pm 1.04$ & $3.56 \pm 0.92$ & $4.71 \pm 0.98$ \\
\hline & 18:1-22:6 (diacyl) & $1.80 \pm 0.39$ & $1.58 \pm 0.18$ & $1.44 \pm 0.79$ & $1.23 \pm 0.15$ & $1.26 \pm 0.41$ & $1.55 \pm 0.29$ \\
\hline & 20:0-22:6 (diacyl) & $1.74 \pm 0.29$ & $2.36 \pm 0.49$ & $1.00 \pm 0.45$ & $1.65 \pm 0.45$ & $1.63 \pm 0.36$ & $1.69 \pm 0.45$ \\
\hline & 22:4-22:6 (diacyl) & $7.68 \pm 2.36$ & $5.82 \pm 1.23$ & $3.02 \pm 1.03$ & $1.21 \pm 0.25$ & $1.16 \pm 0.30$ & $1.37 \pm 0.22$ \\
\hline PS_AA_DHA & 20:4-22:6 (diacyl) & $2.33 \pm 0.40$ & $2.51 \pm 0.32$ & $1.13 \pm 0.36$ & $1.54 \pm 0.49$ & $1.14 \pm 0.34$ & $1.22 \pm 0.25$ \\
\hline Lyso PI_AA & 20:4 (acyl) & $2.80 \pm 0.19$ & $3.27 \pm 0.32$ & $2.26 \pm 0.73$ & $1.33 \pm 0.22$ & $1.13 \pm 0.31$ & $1.34 \pm 0.30$ \\
\hline \multirow[t]{5}{*}{ PI_AA_acyl } & 16:1-20:4 (diacyl) & $1.88 \pm 0.47$ & $1.31 \pm 0.08$ & $2.11 \pm 0.65$ & $1.71 \pm 0.11$ & $1.49 \pm 0.42$ & $1.67 \pm 0.43$ \\
\hline & $16: 0-20: 4$ (diacyl) & $2.08 \pm 0.43$ & $1.88 \pm 0.37$ & $0.65 \pm 0.18^{*}$ & $1.32 \pm 0.22$ & $0.94 \pm 0.21$ & $1.32 \pm 0.19$ \\
\hline & 18:0-20:4 (alkyl_acyl) & $2.13 \pm 0.37$ & $2.63 \pm 0.45$ & $1.02 \pm 0.24$ & $1.25 \pm 0.15$ & $1.17 \pm 0.31$ & $1.23 \pm 0.25$ \\
\hline & 18:1-20:4 (diacyl) & $2.43 \pm 0.24$ & $2.27 \pm 0.33$ & $1.30 \pm 0.35$ & $1.11 \pm 0.23$ & $0.95 \pm 0.30$ & $1.11 \pm 0.33$ \\
\hline & 18:0-20:4 (diacyl) & $2.41 \pm 0.38$ & $2.25 \pm 0.35$ & $1.11 \pm 0.20$ & $1.84 \pm 0.22$ & $1.49 \pm 0.32$ & $1.70 \pm 0.30$ \\
\hline PI_DHA_acyl & 18:0-22:6 (diacyl) & $1.64 \pm 0.12$ & $1.75 \pm 0.29$ & $0.73 \pm 0.23$ & $1.96 \pm 0.16$ & $1.43 \pm 0.58$ & $1.54 \pm 0.17$ \\
\hline
\end{tabular}

${ }^{a}$ Data are mean \pm SD.

${ }^{*} p<0.05$.

$d=2.51$, respectively), whereas those of lyso-PE (18:1), lyso-PE (22:6), and lyso-PE (22:4) were decreased in the CPu of that group $(p=0.039, p=0.033$, and $p=0.026$, and $d=2.13, d=$ 2.55 , and $d=2.42$, respectively; Fig. $6 F)$. To verify that increased PE (18:0p-22:6) in the PFC was not ascribed to PE incorporated into the blood cells from liposomes inside blood vessels, PE (18: 0p-22:6) in blood cells was measured by MALDI-IMS. The ratio of PE (18:0p-22:6) to total lipids for the PEL group did not differ from that for the CL group (Fig. 6G). Thus, PE (18:0p-22:6) was incorporated at least into the PFC, but not blood cells, after the injection of PE liposomes. Similar to prenatal undernutrition, PE (18:0p-22:6) injection did not increase the amount of other PE, PS, and PI containing DHA or AA (Fig. 6F; Table 5). Furthermore, most phospholipids that varied in amount after exposure to prenatal undernutrition were not altered by PEL injection, although lyso PE (22:6) was reduced in both 40F offspring and PEL-injected rats (Figs. 5D, 6F; Table 5).

\section{Discussion}

The findings of our study suggest that changes in phospholipid composition led by prenatal undernutrition is associated with hyperactivity in rats, and plasmalogen PE (18:0p-22:6) injection reproduces a part of hyperactive behaviors. Regarding the cell membrane, plasmalogens constitute $\sim 20 \%$ of total phospholipids, both in the rat cerebral cortex and the human brain (Braverman and Moser, 2012). In humans, ethanolamine plasmalogen constitutes $57 \%$ and $84 \%$ of the glycerophosphoethanolamine fraction of the gray and white matter of the frontal cortex, respectively (Braverman and Moser, 2012). Neurons and myelin are rich in plasmalogens, which decrease membrane fluidity, increase membrane rigidity, and allow tight packing of phospholipids in the membrane (Dean and Lodhi, 2018). Plasmalogens play a role in membrane trafficking and fusion processes, Schwann cell differentiation and function, molecule antioxidation, and inhibition of neuronal apoptotic signaling. Hence, a deficiency of plasmalogens induces impairments of neurotransmitter release from synaptosomes to the presynaptic cleft, myelination and axonal sorting by Schwann cells, and neuronal apoptosis signaling (Dean and Lodhi, 2018). These reports suggest the clinical importance of plasmalogens to the nervous system. The amyloid $\beta$ peptide, which is rich in the brains of patients with Alzheimer's disease, reduces AGPS protein stability and decreases plasmalogen PE levels in patients with Alzheimer's disease (Han et al., 2001; Grimm et al., 2011). Recently, Hossain et al. (2017) reported that inflammatory stimuli, such as the administration of lipopolysaccharide or polyriboinosinic:polyribocytidylic acid, reduce plasmalogens in murine glial cells through the activation of NF- $\kappa \mathrm{B}$, which downregulates Gnpat through increased c-Myc recruitment to the Gnpat promoter. Similar findings have been observed for the murine brain after aging, exposure to chronic restraint stress, and injection of lipopolysaccharide; furthermore, 
the reduction of plasmalogen-induced activation of microglial cells and elevated expression of proinflammatory cytokines (Hossain et al., 2017). In brains from transgenic mice model of Alzheimer's disease, and postmortem brain tissues from patients with Alzheimer's disease, Gnpat reduction via a similar mechanism has been observed (Hossain et al., 2017). Likewise, maternal infection, obesity, a high-fat diet, and restraint stress with brightlight exposure causes microglial cell activation and proinflammatory cytokine induction in the fetal and postnatal brain of rodents and monkeys, and maternal stress results in anxiety-like, depressive, and aggressive behavior, and schizophrenia-like behavior in offspring (Bilbo and Tsang, 2010; Grayson et al., 2010; Matrisciano et al., 2012; Diz-Chaves et al., 2013; Sasaki et al., 2013; Marques et al., 2015). In addition, activation of microglia is augmented in the brain, including the anterior and orbitofrontal cortices in young adults with ASD, although the distribution pattern of activated microglia is similar to that of healthy control subjects, as determined by positron emission tomography (Suzuki et al., 2013). Maternal obesity before pregnancy is considered a risk factor for ADHD and ASD in humans (Andersen et al., 2018). Obesity is involved in elevated inflammatory mediators (e.g., IL-6), which induces Th17 cell differentiation. IL-17A secreted from Th17 may act to promote ASD by affecting fetal neurodevelopment (Wong and Hoeffer, 2018). These results suggest that brain inflammation plays a key role in behavior and that plasmalogen alters brain function through its anti-inflammatory effects. However, in our study, microglial cell activation was not altered by maternal undernutrition, at least for the adult offspring. Further, injection of plasmalogen PE (18:0p-22:6) to adult rats in the $40 \mathrm{~F}$ group altered the phospholipid composition and resulted in two characteristic behaviors: frequent crossing and long time spent in the center area in the open-field test.

Therefore, the hyperactivity of the rat offspring that were exposed to prenatal undernutrition may be attributable to the phospholipid composition of the brain rather than a direct effect of undernutrition on inflammatory reactions. Patients with rhizomelic chondrodysplasia punctata (RCDP), who display plasmalogen deficiency, have psychomotor retardation; and in severely affected cases, they display microcephaly and cerebellar atrophy (Berger et al., 2016). Myelination and neuronal migration are thought to be causes of these features of patients with RCDP (Berger et al., 2016). RCDP Type 1, Type 2, and Type 3 are caused by mutations of PEX7, GNPAT, and AGPS (Berger et al., 2016), respectively, all of which contribute to plasmalogen synthesis. In our study, expression of the latter two genes was elevated in the PFC. In RCDP fibroblasts with the PEX7 mutation, peroxisome targeting signal 2 protein, phytanoyl-CoA hydroxylase, and AGPS fail to be imported into the peroxisome (Yu et al., 2013). The PEX7 homozygous mutation has also been found in three ASD children whose unaffected siblings were heterozygous or WT within one family (Yu et al., 2013). Moreover, single nucleotide polymorphism fine mapping has shown that GNPAT is a candidate gene for schizophrenia, as is DISC1 (Liu et al., 2006). These findings suggest that altered phospholipid metabolism, especially plasmalogen metabolism, may be involved in a person's vulnerability to developmental and psychiatric disorders. Gnpat-KO mice show delayed migration of granule cell precursors, enhanced apoptosis in the cerebellum, and hypomyelination and dysmyelination in the neocortex, cerebellum, and corpus callosum (Berger et al., 2016). Aberrant myelination may be one of the key factors in hyperactivity because MR findings suggest altered myelination in the white matter of adults with ADHD (Wu et al., 2017). Skin fibroblasts derived from patients with RCDP showed reduced PlsEtn, whereas the total amount of PE was maintained by an increase in other PEs (Dorninger et al., 2015). Polyunsaturated fatty acid (PUFA)-containing PlsEtn was reduced in these cells, and AA-containing, but not DHAcontaining, PE species mainly compensated for PlsEtn deficiency (Dorninger et al., 2015). Similar findings have been observed for Gnpat-KO mice; therefore, the ratio among essential PUFAs, as well as the ratio between PlsEtn and PE, may be critical for brain development, and a shift in these ratios may be the cause of the psychomotor retardation of patients with RCDP. In contrast, in our study, the levels of PE (18:0p-22:6) in the PFC and lyso PE (22:6) in $\mathrm{CPu}$ were both altered by exposure to prenatal undernutrition and PE (18:0p-22:6) injection. Additionally, injection of POPE, which is not a plasmalogen, did not alter rat behavior. A specific plasmalogen, such as PE (18:0p-22:6), and a specific DHA-containing PE, such as lyso PE (22:6), coupled with the level of plasmalogen in the brain, may have a function for behavior. Exogenous administration of plasmalogen can be considered as a potential therapeutic strategy as it results in changes in the phospholipid composition of the brain. Regarding psychiatric disorders, the levels of PUFAs (e.g., PE22:5n6, PC20:3n6, and PC22:5n6) were lower in the white matter adjacent to the dorsolateral PFC of a patient with schizophrenia, whereas the level of PE20:2n6 was higher, and those of PE22:5n6, PC20:4n6, and PC22:5n6 were lower, in the white matter of the patient with bipolar disorder, despite no alterations in the plasmalogen level for both disorders (Ghosh et al., 2017). A subset combination of the head group (e.g., ethanolamine, choline, serine, or inositol) and a fatty acid tail, such as DHA or AA, which are incorporated into the phospholipid, may be critical to evoke the behavioral alterations that are characteristic of developmental or psychiatric disorders, and thus, could be a therapeutic target to improve conditions for patients with these diseases. As in one of the trials, the administration of a PS supplement in a chewable tablet presentation improved ADHD for children 4-14 years of age (Hirayama et al., 2014). Exogenous PS can cross the blood-brain barrier and function in the brain (Glade and Smith, 2015). Regarding the delivery of plasmalogen into the brain across the blood-brain barrier, liposomes with PC may be one of the preferred carriers because the intravenous injection of liposomes has been the preferred delivery route for many previous studies (Vieira and Gamarra, 2016). However, PE has a cationic head, and the liposome may not be able to reach the brain because of nonspecific binding to the peripheral tissues and serum proteins (Vieira and Gamarra, 2016) if the ethanolamine head is exposed on the surface of the liposome membrane. In our study, the amount of PE (18:0p-22:6) was not altered in blood cells, suggesting that little of the injected PE was taken into the peripheral tissues during circulation in the brain before liposomes were captured by the liver. Plasmalogen is asymmetrically localized to the inner leaflet of the myelin membrane bilayer (Kirschner and Ganser, 1982), and plasmalogen-rich membranes tend to form nonlamellar inverse hexagonal structures compared with the membrane, which exclusively consists of diacyl phospholipids (Dean and Lodhi, 2018). The surface of the liposomes that were used in our study may be electrically neutral, and their nonspecific binding to the peripheral tissues and serum proteins may be prevented.

In conclusion, maternal undernutrition during early pregnancy led to the hyperactivity of male rat offspring, and the behavioral changes observed may be, in part, caused by an alteration of the plasmalogen composition in the PFC, which was induced by the activation of the phospholipid synthetic pathway. 
Ethanolamine plasmalogen (18:0p-22:6) appears to play a critical role in behavior. Thus, plasmalogens could be candidate therapeutic molecules for improving behavioral disorders. Further study of their complex functions is warranted.

\section{References}

Aleman A, Kahn RS, Selten JP (2003) Sex differences in the risk of schizophrenia: evidence from meta-analysis. Arch Gen Psychiatry 60:565-571.

Amaral AC, Jakovcevski M, McGaughy JA, Calderwood SK, Mokler DJ, Rushmore RJ, Galler JR, Akbarian SA, Rosene DL (2015) Prenatal protein malnutrition decreases KCNJ3 and 2DG activity in rat prefrontal cortex. Neuroscience 286:79-86.

Andersen CH, Thomsen PH, Nohr EA, Lemcke S (2018) Maternal body mass index before pregnancy as a risk factor for ADHD and autism in children. Eur Child Adolesc Psychiatry 27:139-148.

Arnett AB, Pennington BF, Willcutt EG, DeFries JC, Olson RK (2015) Sex differences in ADHD symptom severity. J Child Psychol Psychiatry 56:632-639.

Badaut J, Regli L (2004) Distribution and possible roles of aquaporin 9 in the brain. Neuroscience 129:971-981.

Benjamins J, Murphy E, Seyfried T (2011) Lipids. In: Basic neurochemistry: principles of molecular, cellular, and medical neurobiology, Ed 8 (Brady S, Siegel G, Albers RW, Price D, eds), pp 81-100. Amsterdam: Elsevier.

Berger J, Dorninger F, Forss-Petter S, Kunze M (2016) Peroxisomes in brain development and function. Biochim Biophys Acta 1863:934-955.

Bilbo SD, Tsang V (2010) Enduring consequences of maternal obesity for brain inflammation and behavior of offspring. FASEB J 24:2104-2115.

Braverman NE, Moser AB (2012) Functions of plasmalogen lipids in health and disease. Biochim Biophys Acta 1822:1442-1452.

Brown AS, Susser ES (2008) Prenatal nutritional deficiency and risk of adult schizophrenia. Schizophr Bull 34:1054-1063.

Bystron I, Blakemore C, Rakic P (2008) Development of the human cerebral cortex: Boulder Committee revisited. Nat Rev Neurosci 9:110-122.

Dahoun T, Trossbach SV, Brandon NJ, Korth C, Howes OD (2017) The impact of disrupted-in-schizophrenia 1 (DISC1) on the dopaminergic system: a systematic review. Transl Psychiatry 7:e1015.

Dean JM, Lodhi IJ (2018) Structural and functional roles of ether lipids. Protein Cell 9:196-206.

Diz-Chaves Y, Astiz M, Bellini MJ, Garcia-Segura LM (2013) Prenatal stress increases the expression of proinflammatory cytokines and exacerbates the inflammatory response to LPS in the hippocampal formation of adult male mice. Brain Behav Immun 28:196-206.

Dorninger F, Brodde A, Braverman NE, Moser AB, Just WW, Forss-Petter S, Brügger B, Berger J (2015) Homeostasis of phospholipids: the level of phosphatidylethanolamine tightly adapts to changes in ethanolamine plasmalogens. Biochim Biophys Acta 1851:117-128.

Erb C (2006) Embryology and teratology. In: The laboratory rat, Ed 2 (Suckow MA, Franklin SH, Craig L, eds), pp 817-846. Amsterdam: Elsevier.

Fineberg AM, Ellman LM, Schaefer CA, Maxwell SD, Shen L, Chaudhury NH, Cook AL, Bresnahan MA, Susser ES, Brown AS (2016) Fetal exposure to maternal stress and risk for schizophrenia spectrum disorders among offspring: differential influences of fetal sex. Psychiatry Res 236:91-97.

Fritts ME, Asbury ET, Horton JE, Isaac WL (1998) Medial prefrontal lesion deficits involving or sparing the prelimbic area in the rat. Physiol Behav 64:373-380.

Ghosh S, Dyer RA, Beasley CL (2017) Evidence for altered cell membrane lipid composition in postmortem prefrontal white matter in bipolar disorder and schizophrenia. J Psychiatr Res 95:135-142.

Glade MJ, Smith K (2015) Phosphatidylserine and the human brain. Nutrition 31:781-786.

Götz M, Huttner WB (2005) The cell biology of neurogenesis. Nat Rev Mol Cell Biol 6:777-788.

Grayson BE, Levasseur PR, Williams SM, Smith MS, Marks DL, Grove KL (2010) Changes in melanocortin expression and inflammatory pathways in fetal offspring of nonhuman primates fed a high-fat diet. Endocrinology 151:1622-1632.

Grimm MO, Kuchenbecker J, Rothhaar TL, Grösgen S, Hundsdörfer B, Burg VK, Friess P, Müller U, Grimm HS, Riemenschneider M, Hartmann T (2011) Plasmalogen synthesis is regulated via alkyl-dihydroxyacetonephosphate-synthase by amyloid precursor protein processing and is affected in Alzheimer's disease. J Neurochem 116:916-925.
Han X, Holtzman DM, McKeel DW Jr (2001) Plasmalogen deficiency in early Alzheimer's disease subjects and in animal models: molecular characterization using electrospray ionization mass spectrometry. J Neurochem 77:1168-1180.

Hino K, Kimura T, Udagawa J (2019) Handling has an anxiolytic effect that is not affected by the inhibition of the protein kinase $C$ pathway in adult prenatal undernourished male rat offspring. Congenit Anom (Kyoto). Advance online publication. Retrieved March 18, 2019. doi: $10.1111 /$ cga. 12332.

Hirayama S, Terasawa K, Rabeler R, Hirayama T, Inoue T, Tatsumi Y, Purpura M, Jäger R (2014) The effect of phosphatidylserine administration on memory and symptoms of attention-deficit hyperactivity disorder: a randomised, double-blind, placebo-controlled clinical trial. J Hum Nutr Diet 27:284-291.

Hoek HW, Brown AS, Susser E (1998) The Dutch famine and schizophrenia spectrum disorders. Soc Psychiatry Psychiatr Epidemiol 33:373-379.

Honsho M, Fujiki Y (2017) Plasmalogen homeostasis: regulation of plasmalogen biosynthesis and its physiological consequence in mammals. FEBS Lett 591:2720-2729.

Hossain MS, Abe Y, Ali F, Youssef M, Honsho M, Fujiki Y, Katafuchi T (2017) Reduction of ether-type glycerophospholipids, plasmalogens, by NF- $\kappa$ B signal leading to microglial activation. J Neurosci 37:4074-4092.

Hossen MA, Nagata Y, Waki M, Ide Y, Takei S, Fukano H, Romero-Perez GA, Tajima S, Yao I, Ohnishi K, Setou M (2015) Decreased level of phosphatidylcholine (16:0/20:4) in multiple myeloma cells compared to plasma cells: a single-cell MALDI-IMS approach. Anal Bioanal Chem 407:5273-5280.

Jenkins BT, Hajra AK (1976) Glycerol kinase and dihydroxyacetone kinase in rat brain. J Neurochem 26:377-385.

Jinks AL, McGregor IS (1997) Modulation of anxiety-related behaviours following lesions of the prelimbic or infralimbic cortex in the rat. Brain Res 772:181-190

Khashan AS, Abel KM, McNamee R, Pedersen MG, Webb RT, Baker PN, Kenny LC, Mortensen PB (2008) Higher risk of offspring schizophrenia following antenatal maternal exposure to severe adverse life events. Arch Gen Psychiatry 65:146-152.

Kimura T, Hino K, Kono T, Takano A, Nitta N, Ushio N, Hino S, Takase R, Kudo M, Daigo Y, Morita W, Nakao M, Nakatsukasa M, Tamagawa T, Rafiq AM, Matsumoto A, Otani H, Udagawa J (2018) Maternal undernutrition during early pregnancy inhibits postnatal growth of the tibia in the female offspring of rats by alteration of chondrogenesis. Gen Comp Endocrinol 260:58-66.

Kinney DK, Miller AM, Crowley DJ, Huang E, Gerber E (2008) Autism prevalence following prenatal exposure to hurricanes and tropical storms in Louisiana. J Autism Dev Disord 38:481-488.

Kirschner DA, Ganser AL (1982) Myelin labeled with mercuric chloride: asymmetric localization of phosphatidylethanolamine plasmalogen. J Mol Biol 157:635-658.

Kundakovic M (2014) Chapter 24: DNA methyltransferase inhibitors and psychiatric disorders A2. In: Epigenetics in psychiatry (Grayson DR, Avramopoulos D, eds), pp 497-514. Amsterdam: Elsevier.

Kundakovic M, Jaric I (2017) The epigenetic link between prenatal adverse environments and neurodevelopmental disorders. Genes (Basel) 8:E104.

Liao F, Yoon H, Kim J (2017) Apolipoprotein E metabolism and functions in brain and its role in Alzheimer's disease. Curr Opin Lipidol 28:60-67.

Liu YL, Fann CS, Liu CM, Chen WJ, Wu JY, Hung SI, Chen CH, Jou YS, Liu SK, Hwang TJ, Hsieh MH, Ouyang WC, Chan HY, Chen JJ, Yang WC, Lin CY, Lee SF, Hwu HG (2006) A single nucleotide polymorphism fine mapping study of chromosome 1q42.1 reveals the vulnerability genes for schizophrenia, GNPAT and DISC1: association with impairment of sustained attention. Biol Psychiatry 60:554-562.

Markham JA, Koenig JI (2011) Prenatal stress: role in psychotic and depressive diseases. Psychopharmacology 214:89-106.

Marques AH, Bjørke-Monsen AL, Teixeira AL, Silverman MN (2015) Maternal stress, nutrition and physical activity: impact on immune function, CNS development and psychopathology. Brain Res 1617:28-46.

Matrisciano F, Tueting P, Maccari S, Nicoletti F, Guidotti A (2012) Pharmacological activation of group-II metabotropic glutamate receptors corrects a schizophrenia-like phenotype induced by prenatal stress in mice. Neuropsychopharmacology 37:929-938.

Matrisciano F, Tueting P, Dalal I, Kadriu B, Grayson DR, Davis JM, Nicoletti 
F, Guidotti A (2013) Epigenetic modifications of GABAergic interneurons are associated with the schizophrenia-like phenotype induced by prenatal stress in mice. Neuropharmacology 68:184-194.

McKenna M, Dienel G, Sonnewald U, Waagepetersen H, Schousboe A (2011) Energy metabolism of the brain. In: Basic neurochemistry: principles of molecular, cellular, and medical neurobiology (Brady S, Siegel G, Albers RW, Price D, eds), pp 200-231. Amsterdam: Elsevier.

Moreno M, Economidou D, Mar AC, López-Granero C, Caprioli D, Theobald DE, Fernando A, Newman AH, Robbins TW, Dalley JW (2013) Divergent effects of $\mathrm{D}_{2} / 3$ receptor activation in the nucleus accumbens core and shell on impulsivity and locomotor activity in high and low impulsive rats. Psychopharmacology 228:19-30.

Morita SY, Deharu Y, Takata E, Nakano M, Handa T (2008) Cytotoxicity of lipid-free apolipoprotein B. Biochim Biophys Acta 1778:2594-2603.

Nguyen LN, Ma D, Shui G, Wong P, Cazenave-Gassiot A, Zhang X, Wenk MR, Goh EL, Silver DL (2014) Mfsd2a is a transporter for the essential omega-3 fatty acid docosahexaenoic acid. Nature 509:503-506.

Nishiumi S, Kobayashi T, Ikeda A, Yoshie T, Kibi M, Izumi Y, Okuno T, Hayashi N, Kawano S, Takenawa T, Azuma T, Yoshida M (2012) A novel serum metabolomics-based diagnostic approach for colorectal cancer. PLoS One 7:e40459.

Roseboom TJ, van der Meulen JH, Ravelli AC, Osmond C, Barker DJ, Bleker OP (2001) Effects of prenatal exposure to the Dutch famine on adult disease in later life: an overview. Mol Cell Endocrinol 185:93-98.

Sasaki A, de Vega WC, St-Cyr S, Pan P, McGowan PO (2013) Perinatal high fat diet alters glucocorticoid signaling and anxiety behavior in adulthood. Neuroscience 240:1-12.

Schoenwolf GC, Bleyl SB, Brauer PR, Francis-West PH, Larsen WJ (2015) Larsen's human embryology, Ed 5. Amsterdam: Elsevier.

Scofield MD, Heinsbroek JA, Gipson CD, Kupchik YM, Spencer S, Smith AC, Roberts-Wolfe D, Kalivas PW (2016) The nucleus accumbens: mechanisms of addiction across drug classes reflect the importance of glutamate homeostasis. Pharmacol Rev 68:816-871.

Spencer RC, Devilbiss DM, Berridge CW (2015) The cognition-enhancing effects of psychostimulants involve direct action in the prefrontal cortex. Biol Psychiatry 77:940-950.

Sugiyama E, Masaki N, Matsushita S, Setou M (2015) Ammonium sulfate improves detection of hydrophilic quaternary ammonium compounds through decreased ion suppression in matrix-assisted laser desorption/ ionization imaging mass spectrometry. Anal Chem 87:11176-11181.

Suzuki K, Sugihara G, Ouchi Y, Nakamura K, Futatsubashi M, Takebayashi K, Yoshihara Y, Omata K, Matsumoto K, Tsuchiya KJ, Iwata Y, Tsujii M, Sugiyama T, Mori N (2013) Microglial activation in young adults with autism spectrum disorder. JAMA Psychiatry 70:49-58.

Taguchi R, Ishikawa M (2010) Precise and global identification of phospholipid molecular species by an orbitrap mass spectrometer and automated search engine lipid search. J Chromatogr A 1217:4229-4239.

Tsugawa H, Bamba T, Shinohara M, Nishiumi S, Yoshida M, Fukusaki E (2011) Practical non-targeted gas chromatography/mass spectrometrybased metabolomics platform for metabolic phenotype analysis. J Biosci Bioeng 112:292-298.

Vance JE (2015) Phospholipid synthesis and transport in mammalian cells. Traffic 16:1-18.

Vieira DB, Gamarra LF (2016) Getting into the brain: liposome-based strategies for effective drug delivery across the blood-brain barrier. Int J Nanomed 11:5381-5414.

Werling DM, Geschwind DH (2013) Sex differences in autism spectrum disorders. Curr Opin Neurol 26:146-153.

Wong H, Hoeffer C (2018) Maternal IL-17A in autism. Exp Neurol 299:228-240

Wu ZM, Bralten J, Cao QJ, Hoogman M, Zwiers MP, An L, Sun L, Yang L, Zang YF, Franke B, Wang YF (2017) White matter microstructural alterations in children with ADHD: categorical and dimensional perspectives. Neuropsychopharmacology 42:572-580.

Yeagle PL (2016) Biogenesis of membrane lipids. In: The membranes of cells, Ed 3, pp 57-71. Boston: Academic.

Yu TW, Chahrour MH, Coulter ME, Jiralerspong S, Okamura-Ikeda K, Ataman B, Schmitz-Abe K, Harmin DA, Adli M, Malik AN, D'Gama AM, Lim ET, Sanders SJ, Mochida GH, Partlow JN, Sunu CM, Felie JM, Rodriguez J, Nasir RH, Ware J, et al. (2013) Using whole-exome sequencing to identify inherited causes of autism. Neuron 77:259-273.

Zemski Berry KA, Gordon WC, Murphy RC, Bazan NG (2014) Spatial organization of lipids in the human retina and optic nerve by MALDI imaging mass spectrometry. J Lipid Res 55:504-515.

Zhu Y, Yang D, Ji W, Huang T, Xue L, Jiang X, Chen L, Wang F (2016) The relationship between neurocircuitry dysfunctions and attention deficit hyperactivity disorder: a review. Biomed Res Int 2016:3821579. 